Pure and Applied Mathematics Quarterly

Volume 9, Number 4

(Special Issue: In memory of

Andrey Todorov, Part 1 of 3 )

$665-738,2013$

\title{
Weak Coupling, Degeneration and Log Calabi-Yau Spaces
}

\author{
Ron Donagi, Sheldon Katz and Martijn Wijnholt
}

\begin{abstract}
We establish a new weak coupling limit in $F$-theory. The new limit may be thought of as the process in which a local model bubbles off from the rest of the Calabi-Yau. The construction comes with a small deformation parameter $t$ such that computations in the local model become exact as $t \rightarrow 0$. More generally, we advocate a modular approach where compact Calabi-Yau geometries are obtained by gluing together local pieces (log Calabi-Yau spaces) into a normal crossing variety and smoothing, in analogy with a similar cutting and gluing approach to topological field theories. We further argue for a holographic relation between $F$-theory on a degenerate Calabi-Yau and a dual theory on its boundary, which fits nicely with the gluing construction.
\end{abstract}

Keywords: String compactification, F-theory, Calabi-Yau, elliptic fibration, semi-stable degeneration, smoothing, variation of Hodge structure.

Received February 7, 2013. 


\section{Contents}

1 Introduction 667

2 Local and global models 670

2.1 Semi-stable degeneration . . . . . . . . . . . . . . . 6 670

2.2 Normal cone degeneration . . . . . . . . . . . . . . . . 673

2.3 Degenerating the elliptic fibration . . . . . . . . . . . 675

2.4 Caveats . . . . . . . . . . . . . . . . 680

3 Gluing a Calabi-Yau from local pieces 681

3.1 Some aspects of topological field theories . . . . . . . . . . . . 681

3.2 Analogy between topological and holomorphic field theories . . . . 684

3.3 Smoothing criteria of Kawamata/Namikawa . . . . . . . . . . . 691

3.4 Criteria for the existence of an elliptic fibration . . . . . . . . 694

3.5 Example: bubbling off an $S U(5)_{G U T}$ model $\ldots \ldots \ldots$. . . . . 697

3.6 The general case . . . . . . . . . . . . . . 700

4 The form of the Lagrangian in a degeneration limit $\quad \mathbf{7 0 2}$

4.1 Asymptotics from monodromy . . . . . . . . . . . . . 703

4.2 The limiting mixed Hodge structure _ . . . . . . . . . . 705

4.3 Asymptotic form of the superpotential . . . . . . . . . . 708

4.4 The Kähler potential . . . . . . . . . . . . . . . . . . . 717

4.5 Euler character . . . . . . . . . . . . . . . . . . . 719

4.6 Bulk versus boundary . . . . . . . . . . . . . . 720

A Mixed Hodge structures and monodromy 
B On the value of the holomorphic Chern-Simons action

\section{Introduction}

In type II string theories, gauge and matter fields are localized on submanifolds of the ten-dimensional space-time. Therefore the study of type II compactifications (or their $M / F$-theory versions) usually proceeds by splitting the analysis in two steps: first one studies a local model in detail, and then one tries to embed it in a global UV completion. Furthermore, it has been suggested that the hierarchy between the weak scale and the Planck scale, or between the GUT scale and the Planck scale, make such a two-step procedure natural. This raises the important question: what is the precise relationship between the local model and the global model?

One expects that a local model will arise as a limit of a global model. In [1] a decoupling limit was defined by taking the ratio of the volume of the divisor supporting the Standard Model and the volume of the total space to zero. In $F$-theory, this was studied in detail in $[2,3]$. However, this is a limit in the Kähler moduli space, which has no effect on the complex structure of a global model. In the holomorphic world, functions are determined by their behaviour on a small neighbourhood, so 'infinity does not decouple.'

We can also see this at the level of the effective action. The $4 d N=1$ supergravity is characterized by a Kähler potential $\mathcal{K}$, a superpotential $W$ and a gauge kinetic function $f$. Non-renormalization theorems restrict the dependence of $W$ and $f$ on the Kähler moduli. As a result, they are largely unchanged in the limit above, and remember the structure of the global model.

In the present paper, we will address this question more systematically for F-theory models, although our ideas should also apply to other settings, such as the situation considered in [1]. From the above discussion it is clear that we need to take a limit to a boundary of the complex structure moduli space. But this must be done carefully, for otherwise the relation between the local and the global model will be very hard to understand. We will see that one can bubble off local models from global models by semi-stable degeneration. In particular, we will define a new type of semi-stable degeneration in $F$-theory which recovers the 
local models studied in [2] from a global model. (For earlier work, see $[4,5,6]$.) If the global model admits a K3 fibration, then we recover a known semi-stable degeneration of the K3 fibers. However, our method also applies if there is no K3 fibration.

The main purpose of this paper however is to place the results of section 2 and reference [7] in a broader perspective. Namely we would like to discuss the roles played by degeneration and its converse, which is a nice way to construct global models from local pieces, as a cutting-and-gluing procedure in holomorphic field theories.

To explain this, let us first define the notion of a log Calabi-Yau space, since this has not yet percolated to the physics literature and it is one of the fundamental concepts studied in this paper. We define a log Calabi-Yau to be a pair $(X, D)$, where $X$ is a variety and $D$ is an effective divisor in $X$ such that the log canonical class $K_{(X, D)} \equiv K_{X}+D$ vanishes. It admits a unique $(n, 0)$-form which is is holomorphic on $X \backslash D$ and has a logarithmic pole along $D$, whose residue is the holomorphic $(n-1)$-form of $D$. A log Calabi-Yau should be thought of as the holomorphic analogue of a real and oriented manifold with boundary.

Now we start with a disconnected sum of Calabi-Yau manifolds with boundary, say $\left(X_{1}, D\right)$ and $\left(X_{2}, D\right)$ in the simplest case. Then we glue these pieces together along the boundaries into a normal crossing variety

$$
Y_{0}=X_{1} \cup_{D} X_{2}
$$

Finally when $Y_{0}$ has a $\log$ structure and satisfies other mild hypotheses, we can deform $Y_{0}$ to a smooth global model $Y_{t}$, where $t \in \mathbf{C}$ is a parameter, by the fundamental smoothing theorem of Kawamata and Namikawa [8]. Obviously this construction comes with a small parameter and a built-in degeneration limit.

In order to make use of this technique in $F$-theory, it is crucial to know that when $Y_{0}$ has an elliptic fibration, this continues to hold for the smoothings $Y_{t}$. We posed this as an open problem at a recent meeting on string theory for mathematicians at SCGP. To our delight, János Kollár has now settled this in the affirmative and proved a number of additional results in [9]. We include a discussion of some of his results in section 3.4. 


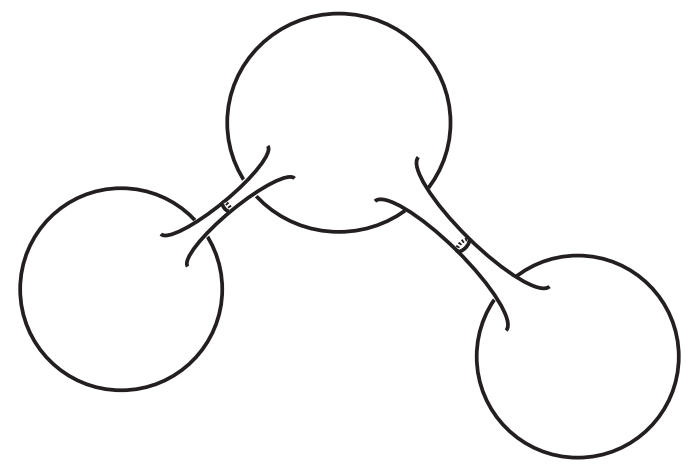

Figure 1: Gluing a Calabi-Yau manifold from local pieces.

Now for physics purposes we are not just interested in complex manifolds per se, but in holomorphic field theory on such a space, that is a theory which depends on the complex structure but not on the Kähler moduli. So in order to use surgery ideas, we want to know how a holomorphic field theory behaves on a family $Y_{t}$. We expect that its partition function and correlators behave nicely in a semi-stable degeneration limit, and that these quantities can in fact be constructed explicitly from the limit as an expansion in $t$.

Furthermore, we expect an interesting interplay between the theory in the bulk and the theory on the boundary. In particular for the case of degeneration in F-theory, we will argue in sections 3 and 4 that computations can also be performed 'holographically' in the boundary theory. We discuss some expected features of the boundary theory and how various known examples fit the pattern. We believe these points give a new perspective on compactifications in which log varieties play a central role, for $F$-theory and more generally for string or field theories on complex spaces.

Coming back to $F$-theory, recall that for $F$-theory compactifications to eight dimensions there are essentially only two weak coupling limits (more precisely, type II degenerations [10]), which yield the $E_{8} \times E_{8}$ and the $S O(32)$ heterotic string. As will hopefully be clear, below eight dimensions there seems to be a veritable zoo of new degeneration limits, and the methods discussed in this paper allow one to construct them. The global-to-local degeneration which splits off a 
local $d P_{9}$-fibration will be our main example in this paper, but much more exotic examples could be considered. A closely related paper [7] examines another new type of degeneration, namely a stable version of the Sen limit, from the general point of view advocated here.

In section 4 we discuss the $t$-dependence of the Lagrangian in a degeneration limit by applying the theory of mixed Hodge structures. Although somewhat abstract, this approach is extremely powerful and allows us to fix much of the physics from very little input. An interesting aspect of this analysis is the interpretation of brane superpotentials as describing an obstruction to splitting the mixed Hodge structure into pure pieces.

Our approach answers several questions that were not clear previously. Regarding the construction of phenomenological GUT models along the lines of [2], we believe that one of the most important issues that are clarified is that there is a new small expansion parameter $t$ in the game, which we identify explicitly, such that holomorphic calculations in the local model become exact in the limit $t \rightarrow 0$. This explains to what extent we can trust the $E_{8}$ picture of local models, and makes the notion of 'dropping the subleading terms' in [2] more precise.

We believe that having such a small modulus is an important step forward in understanding global models, raising various interesting phenomenological questions. Although the net amount of chiral matter cannot change if we continuously vary $t$, more detailed properties of the effective Lagrangian definitely depend on $t$, as seen in section 4 . The behaviour of the Calabi-Yau metric as $t \rightarrow 0$ has not been much investigated, but one expects an anisotropic picture as shown in figure 1 , where points on different local pieces get infinitely separated in the limit. The modulus $t$ may also play a role in the issue of sequestering.

\section{Local and global models}

\subsection{Semi-stable degeneration}

The data of an $F$-theory compactification consists of an elliptically fibered 
Calabi-Yau manifold with section, and a configuration for a three-form field $\mathrm{C}_{3}$ with flux $\mathrm{G}_{4}=d \mathrm{C}_{3}$. In the present section, the focus will be on aspects of the Calabi-Yau geometry. We will typically have in mind elliptic fourfolds $\pi: Y \rightarrow$ $B_{3}$, although the considerations will be more general.

We would like to define global-to-local degenerations for $F$-theory models. By this, we mean we would like to bubble off a four-fold consisting of a $d P_{9}$-fibration over a divisor $S$ in $B_{3}$, which should capture the behaviour of the elliptic fibration $\pi: Y \rightarrow B_{3}$ along $S$. As in [2], we could do a further degeneration along the lines of [11], in order to relate the $d P_{9}$-fibration to a Hitchin system.

Let us consider a flat family $\pi_{\mathcal{Y}}: \mathcal{Y} \rightarrow \Delta$, where $\Delta$ is a disk parametrized by a variable $t$, such that the generic fibers are smooth but the central fiber $\pi_{\mathcal{Y}}^{-1}(0)$ is not. We will actually be interested in fibers with certain types of singularities, which can however be resolved and followed through the degeneration. This caveat will not affect the validity of our techniques however.

In order to get a reasonable limiting behaviour, the degeneration needs to be fairly mild. Specifically, we will be able to achieve our objectives by a semi-stable degeneration. This means that the total space $\mathcal{Y}$ of the family is smooth and the central fiber is reduced and has at most normal crossing singularities. In local coordinates $\left(z_{1}, \ldots, z_{n}\right)$, a normal crossing singularity is a singularity given by an equation of the form $z_{1} \cdots z_{k}=0$.

According to Mumford's semi-stable reduction theorem, any degeneration may be put in the form

$$
z_{1} \cdots z_{k}=t
$$

for small $t$, possibly after a base change (i.e. pulling back the base of the family by an analytic map $\Delta \rightarrow \Delta$ ) and then blowing up and down. A semi-stable degeneration need not be unique, as there may be many different birationally equivalent models. A degeneration is said to be stable if in addition, the central fiber has no infinitesimal automorphisms.

Semi-stable degenerations have a number of nice properties. One of these properties is that they essentially become 'smooth' if we include logarithmic differential forms. Let us denote the fibers $\pi_{\mathcal{Y}}^{-1}(t)$ by $Y_{t}$, and the normal crossing divisor of $Y_{0}$ by $Z$. Suppose that in local coordinates, the equation of $Z$ is given 
by $z_{1} \cdots z_{k}=0$. Let us consider the spaces

$$
\Omega_{Y_{0}}^{q}(\log Z)
$$

of logarithmic differential forms on $Y_{0}$. For $q=1$, this is defined to be the subsheaf of meromorphic differential forms on $Y_{0}$ generated by $d z_{i} / z_{i}$ for $1 \leq i \leq k$ and $d z_{i}$ for $k+1 \leq i \leq n$, together with the relation

$$
d \log \left(z_{1}\right)+\ldots+d \log \left(z_{k}\right)=0
$$

For larger $q$ we just take exterior powers. More generally one can define logarithmic forms with respect to a log structure, but we will not do so here. The exterior derivative takes logarithmic forms to logarithmic forms, so we get a complex and we can compute cohomology. It will be convenient to use the short-hand notation

$$
H_{\log }^{k}\left(Y_{0}\right) \equiv \mathbb{H}^{k}\left(Y_{0}, \Omega_{Y_{0}}^{\bullet}(\log Z)\right)
$$

for the logarithmic de Rham cohomology of $Y_{0}$. The spectral sequence for $\mathbb{H}^{k}$ degenerates at $E_{1}$, so we get the decomposition $H_{\log }^{k}\left(Y_{0}\right)=\sum_{k=p+q} H^{p}\left(\Omega_{Y_{0}}^{q}(\log Z)\right)$ on $Y_{0}$. As motivation for section 3 , suppose that $k=2$ and $W_{1}, W_{2}$ are the components of $Y_{0}$. Then the restriction to $W_{i}$ of $\Omega_{Y_{0}}^{n}(\log Z)$ is just the $\log$ canonical bundle $\Omega_{W_{i}}(Z)$ of the pair $\left(W_{i}, Z\right)$. In particular if $Y_{0}$ is Calabi-Yau in the sense that $\Omega_{Y_{0}}^{n}(\log Z)$ is trivial, then each $\left(W_{i}, Z\right)$ is $\log$ Calabi-Yau.

In a degeneration limit, the ordinary cohomology typically jumps, as some classes on $Y_{t}$ will disappear from the cohomology, and some others may appear. According to the work of Steenbrink [12], the cohomology of logarithmic forms gives a geometric way to realize the 'nearby cocycles' on $Y_{0}$. Concretely, for a normal crossing degeneration the sheaves of differential forms $\Omega_{Y_{t}}^{q}$ naturally fit together into a locally free sheaf $\Omega_{\mathcal{Y} / \Delta}^{q}\left(\log Y_{0}\right)$ of relative forms, whose restriction to the central fiber yields $\Omega_{Y_{0}}^{q}(\log Z)$. Similarly, the sheaves $R^{p} \pi_{\mathcal{Y}_{*}} \Omega_{\mathcal{Y} / \Delta}^{q}\left(\log Y_{0}\right)$ are locally free over the base, i.e. they correspond to holomorphic vector bundles over the base. The fiber over $t \neq 0$ is given by $H^{p}\left(\Omega_{Y_{t}}^{q}\right)$ and the fiber over $t=0$ is given by $H^{p}\left(\Omega_{Y_{0}}^{q}(\log Z)\right)$. In particular, the dimensions of the cohomology groups 
$h^{p}\left(\Omega_{Y_{0}}^{q}(\log Z)\right)$ coincide with the Hodge numbers $h^{p, q}\left(Y_{t}\right)$ of the smooth fibers. As we will see later, these results (and some additional properties discussed in appendix A) are very helpful for elucidating the physics of a semi-stable degeneration.

We will also need to introduce the logarithmic tangent bundle, $T(-\log Z)$, as the dual of the logarithmic cotangent bundle $\Omega^{1}(\log Z)$. In the local coordinates above, $T(-\log Z)$ is generated by $z_{i} \partial / \partial z_{i}$ for $i=1, \ldots, k$ and $\partial / \partial z_{i}$ for $i=k+1, \ldots, n$, together with the relation $\sum_{i=1}^{k} z_{i} \partial / \partial z_{i}=0$. The tangent space to the smoothing component of the deformation space is given by $H^{1}(T(-\log Z))$ [13]. By contracting with the logarithmic $(n, 0)$ form, we can relate this to $H^{1}\left(\Omega^{n-1}(\log Z)\right)$.

Another nice aspect of semi-stable degeneration is that the asymptotic behaviour of the Hodge structure is rather well understood. In particular, we can use the orbit theorems [14] to qualitatively understand the asymptotic behaviour of the Lagrangian in the degeneration limit, as we will discuss later.

\subsection{Normal cone degeneration}

Let us first focus on the base $B_{3}$ without worrying about the elliptic fibration. Suppose that the discriminant locus contains a a smooth divisor $S \subset B_{3}$ of ADE singularities. We would like to be able to 'zoom in' on $S$, i.e. we would like to be able to consider the first order neighbourhood of $S$ in $B_{3}$. There is a construction in algebraic geometry which allows us to do this, namely the degeneration to the normal cone. We will first quickly review this construction and refer the interested reader to [15] for more details. Then in the next subsection, we will show how the elliptic fibration can be extended over the normal cone degeneration of $B_{3}$, yielding a semi-stable degeneration of the elliptic fourfold.

The deformation to the normal cone is constructed as follows. We consider the constant family

$$
\mathcal{B}=B_{3} \times \mathbf{C}
$$

parametrized by $t \in \mathbf{C}$. We have the projection $\pi_{\mathcal{B}}: \mathcal{B} \rightarrow \mathbf{C}$ and all fibers are canonically isomorphic to $B_{3}$. Then we blow up $\mathcal{B}$ along the subvariety 
$S \times\{0\} \subset \mathcal{B}$ to obtain a variety $\tilde{\mathcal{B}}$ with exceptional divisor $E \subset \tilde{\mathcal{B}}$, with $E$ isomorphic to the projectivization $\mathbf{P}\left(\mathcal{O} \oplus N_{S}\right)$ of the normal bundle $N_{S}$ of $S$ in $B_{3}$. Via the projection $\pi_{\tilde{\mathcal{B}}}: \tilde{\mathcal{B}} \rightarrow \mathbf{C}$, we get another family of varieties parametrized by $t$. Let $\tilde{B} \subset \tilde{\mathcal{B}}$ be the proper transform of $B_{3} \times\{0\}$, which projects isomorphically to $B_{3}$ via the composite map $\tilde{\mathcal{B}} \rightarrow \mathcal{B} \rightarrow B_{3}$, where the first map is the map $\rho: \tilde{\mathcal{B}} \rightarrow \mathcal{B}$ which blows down $E \subset \tilde{\mathcal{B}}$ to $S \times\{0\} \subset \mathcal{B}$ and the second map is the projection $\pi_{1}$ of $\mathcal{B}=B_{3} \times \mathbf{C}$ to its first factor $S$.

Let us consider the structure of the family $\tilde{\mathcal{B}}$ in more detail. Obviously for $t \neq 0$, the fiber $\pi_{\tilde{\mathcal{B}}}^{-1}(t)$ is still isomorphic to $B_{3}$. However for $t=0$ we get something interesting. We claim that

$$
B_{0}:=\pi_{\tilde{\mathcal{B}}}^{-1}(0)=E \cup \tilde{B}
$$

Furthermore, these two components are glued in the following way. We can think of $E=\mathbf{P}\left(\mathcal{O} \oplus N_{S}\right)$ as the compactification of the total space of $N_{S}$ by a section $S_{\infty}$ at infinity. In this realization, the zero section of $N_{S}$ is identified with a section $S_{0}$ of $\mathbf{P}\left(\mathcal{O} \oplus N_{S}\right)$. Then the two components are glued by identifying $S_{\infty} \subset \mathbf{P}\left(\mathcal{O} \oplus N_{S}\right)$ with the original divisor $S \subset B_{3} \simeq \tilde{B}$.

Let's check (2.6) and the assertions below it by writing down the defining equations for $B_{0}$ explicitly. The divisor $S$ is the zero locus of a section $s$ of $\mathcal{O}_{B_{3}}(S)$. Thus the locus $S \times\{0\}$ that we are blowing up is given by $s=t=0 .{ }^{1}$ The blowup can be constructed as a hypersurface in the fourfold $\mathbf{P}\left(\mathcal{O}_{B_{3}} \oplus \mathcal{O}_{B_{3}}(S)\right)$, a $\mathbf{P}^{1}$-bundle over $B_{3}$. To partially coordinatize this fourfold, we introduce two additional variables $u$ and $v$, with $(u, v)$ taking values in $\mathcal{O}_{B} \oplus \mathcal{O}_{B}(S)$, and then we identify $(u, v) \sim(\lambda u, \lambda v)$ for any $\lambda \in \mathbf{C}^{*}$. The hypersurface $\tilde{\mathcal{B}} \subset \mathbf{P}\left(\mathcal{O}_{B_{3}} \oplus \mathcal{O}_{B_{3}}(S)\right)$ is given by the standard equation of a blowup

$$
s u-t v=0 .
$$

When $s$ and $t$ are not both zero, we find that $u$ and $v$ are uniquely determined up to the action of $\mathbf{C}^{*}$. When $s=t=0$, we get an extra $\mathbf{P}^{1}$ parametrized by

\footnotetext{
${ }^{1}$ Here we have chosen a global construction of the blowup. Alternatively, one can pick local equations $f_{\alpha}=0$ for $S$ in open sets $U_{\alpha}$ and construct the blowup locally, then glue the patches of the blowup according to how the $f_{\alpha}$ change between patches.
} 
$(u, v)$. So for each point on $S \times\{0\}$, we grow an extra $\mathbf{P}^{1}$, showing that the exceptional divisor $E$ is a $\mathbf{P}^{1}$-bundle over $S$. After restricting to the exceptional divisor $E$, i.e. setting $s=t=0$ in $(2.7)$, we see that $(u, v)$ take values in $\mathbf{P}(\mathcal{O} \oplus$ $N_{S}$ ), since $\mathcal{O}_{B}(S)$ restricts to $N_{S}$ on $S$. This completes the demonstration that $E \simeq \mathbf{P}\left(\mathcal{O} \oplus N_{S}\right)$. The zero section $S_{0}$ is given by $v=0$, and $S_{\infty}$ is given by $u=0$.

The total space $\tilde{\mathcal{B}}$ is smooth, but the fibers of $\pi_{\tilde{\mathcal{B}}}$ are not. For $t \neq 0$, we get a variety isomorphic to $B_{3}$ as noted above. But for $t=0$, we get two components, as the equation (2.7) becomes $s u=0$. Away from the zeroes of $s$, we get $u=0$, hence a copy of $B_{3}$ (with $S$ excised). Away from the zeroes of $u$, we get $s=0$, hence the exceptional divisor (minus $S_{\infty}$ ). So altogether, for $t=0$ we get a normal crossing variety with two components.

Let's now take a closer look at the intersection $E \cap \tilde{B}$ of the two components. As we have seen above, $B_{0}=E \cup \tilde{B}$ is the subvariety of $\tilde{\mathcal{B}}$ defined by $t=s u=0$. The two components of $B_{0}$ are therefore defined by $t=s=0$ and $t=u=0$. Since the former are the equations of $S \times\{0\} \subset \mathcal{B}$, they define the exceptional divisor $E$ after pulling back to $\tilde{\mathcal{B}}$. Since $B_{3} \times\{0\}$ is defined by $t=0$, its proper transform $\tilde{B}$ is given by the remaining component, $t=u=0$. Finally, the components intersect in the curve $E \cap \tilde{B}$ defined by $s=t=u=0$. Viewed as a curve inside $E$ it is defined by $u=0$ hence is identified with $S_{\infty}$. Viewed as a curve inside $\tilde{B}$ it is defined by $s=0$, hence is identified with $S$.

This completes the justification of all of our claims about the geometry of $B_{0}$.

Note that the normal cone degeneration itself is not a complex structure deformation in the usual sense. All the fibers for $t \neq 0$ are isomorphic, and at $t=0$ the fiber $B_{0}$ 'jumps.' There is no monodromy around $t=0$. However, nontrivial monodromy will occur once we put a suitable elliptic fibration over $\tilde{\mathcal{B}}$.

\subsection{Degenerating the elliptic fibration}

Our next task is to identify a degeneration for the full Calabi-Yau, including the elliptic fibration. We will do this by extending the elliptic fibration over the one-parameter family considered in the previous subsection, obtained from deformation to the normal cone of a divisor $S \subset B$. This will give us a family $\mathcal{Y}$, 
with fibers $Y_{t}$, such that

$$
Y_{0}=W_{1} \cup_{Z} W_{2}
$$

where $W_{1}$ is an elliptic fibration over $E$ and $W_{2}$ is an elliptic fibration over $\tilde{B} \simeq B_{3}$. Furthermore, $W_{1}$ will have the structure of a $d P_{9}$-fibration over $S$.

Let $B_{3}$ be a smooth three-dimensional $F$-theory base. We can describe an elliptically fibered Calabi-Yau $Y \rightarrow B_{3}$ in generalized Weierstrass form. Given sections $a_{i} \in H^{0}\left(B_{3},-i K_{B_{3}}\right)$, we can write the equation of an elliptic fibration over $B_{3}$ in the form

$$
y^{2}+a_{1} x y+a_{3} y=x^{3}+a_{2} x^{2}+a_{4} x+a_{6}
$$

If we now replace the $a_{i}$ by $t$-dependent sections $a_{i}(t)$, we will get a family

$$
y^{2}+a_{1}(t) x y+a_{3}(t) y=x^{3}+a_{2}(t) x^{2}+a_{4}(t) x+a_{6}(t)
$$

of elliptically fibered Calabi-Yau fourfolds parametrized by $t$, which we can also view as an elliptically fibered Calabi-Yau fivefold over $\mathcal{B}$. We now want to understand what happens when we pass to the normal cone degeneration by blowing up $S \times\{0\}$. A modification is needed since the blowup spoils the Calabi-Yau condition.

The modification is simple enough: we merely have to ensure that the $a_{i}(t)$ remain sections of $H^{0}\left(\tilde{\mathcal{B}},-i K_{\tilde{\mathcal{B}}}\right)$ after pullback to $\tilde{\mathcal{B}}$ in order to preserve the Calabi-Yau condition. This would make $\mathcal{Y}$ a Calabi-Yau fivefold, elliptically fibered over the fourfold $\tilde{\mathcal{B}}$. Composing with the projection $\pi_{\tilde{\mathcal{B}}}: \tilde{\mathcal{B}} \rightarrow \mathbf{C}$, we see that via $\pi_{\mathcal{Y}}: \mathcal{Y} \rightarrow \mathbf{C}, \mathcal{Y}$ can be viewed as a family of threefolds $Y_{t}$ parametrized by $t \in \mathbf{C}$. Each $Y_{t}$, including $Y_{0}$, is Calabi-Yau by the adjunction formula and the Calabi-Yau property of $\mathcal{Y}$, as the divisor class of $Y_{t}$ is trivial since $Y_{t}$ is the pullback of a point $t \in \mathbf{C}$ by the projection $\pi_{\mathcal{Y}}$.

The formula for the canonical bundle of the blowup gives

$$
K_{\tilde{\mathcal{B}}}=\rho^{*}\left(K_{\mathcal{B}}\right)+E .
$$


from which it follows immediately that

$$
-i K_{\tilde{\mathcal{B}}}=\rho^{*}\left(-i K_{\mathcal{B}}\right)-i E
$$

Then we read off from (2.12) the desired Calabi-Yau condition as the requirement that each $a_{i}(t)$ vanishes to order at least $i$ along $S \times\{0\}$. Given such an $a_{i}(t)$, its pullback to $\tilde{\mathcal{B}}$ vanishes along $E$ with multiplicity at least $i$. Letting $e \in$ $H^{0}\left(\mathcal{O}_{\tilde{\mathcal{B}}}(E)\right)$ be a section vanishing along $E$, we see that we have holomorphic sections

$$
\tilde{a}_{i}(t):=\frac{a_{i}(t)}{e^{i}} \in H^{0}\left(\rho^{*}\left(-i K_{\mathcal{B}}\right)-i E\right)=H^{0}\left(-i K_{\tilde{\mathcal{B}}}\right)
$$

which can be used to construct the desired elliptically fibered $\mathcal{Y} \rightarrow \tilde{\mathcal{B}}$ in the usual way.

This gives the desired family of F-theory models. For $t \neq 0, Y_{t}$ is elliptically fibered over $B_{3}$, but $Y_{0}$ is given by (2.8) as a union of elliptic fibrations $W_{1}$ and $W_{2}$, fibered over the respective components $E$ and $\tilde{B} \simeq B_{3}$ of $B_{0}$.

The intersection $Z$ of $Y_{1}$ and $Y_{2}$ is elliptically fibered over the surface $S=$ $E \cap \tilde{B}$. In fact, $Z$ is an elliptically fibered Calabi-Yau threefold. We just have to check that the $\tilde{a}_{i}(t)$ restrict to sections of $-i K_{S}$ on $S$, and for that it suffices to show that $K_{\tilde{\mathcal{B}}}$ restricts to $K_{S}$ on $S$. But that follows from the adjunction formula: $S=E \cap \tilde{B}$ implies that

$$
K_{S}=\left.\left(K_{\tilde{\mathcal{B}}}+E+\tilde{B}\right)\right|_{S},
$$

while $E+\tilde{B}=B_{0}$ is a fiber, which we have already noted is trivial as a divisor class.

The restriction of $K_{\tilde{\mathcal{B}}}$ to $B_{0}$ is in fact the dualizing sheaf of $B_{0}$, denoted $\omega_{B_{0}}$, which plays the role of the canonical bundle for $B_{0}$. This observation will be useful in Section 3.5 when we glue local models.

We can describe $\omega_{B_{0}}$ in terms of its restriction to the components $\tilde{B}$ and $E$ of $B_{0}$. From (2.11) we read off that

$$
\left.\omega_{B_{0}}\right|_{\tilde{B}}=K_{\tilde{B}}+S
$$


We also have by adjunction that $K_{E}=\left.\left(K_{\tilde{B}}+E\right)\right|_{E}=\left.\omega_{B_{0}}\right|_{E}-S_{\infty}$, hence

$$
\left.\omega_{B_{0}}\right|_{E}=K_{E}+S_{\infty}
$$

In other words, sections of $\omega_{B_{0}}$ on each component can be identified with sections of the canonical bundle with first order poles on the intersection $S$.

The restriction of $K_{\tilde{B}}$ to $B_{t}$ for $t \neq 0$ is just the ordinary canonical bundle of $B_{t}$. In fact, $K_{\tilde{B}}$ is the relative dualizing sheaf $\omega_{\pi}$ of the family $\pi_{\tilde{B}}$.

We will now show that $W_{1}$ has the structure of a $\mathrm{dP}_{9}$ fibration over $S$. Recall that a $\mathrm{dP}_{9}$ arises as an elliptic fibration over $\mathbf{P}^{1}$ constructed from (2.9) with $a_{i} \in H^{0}\left(\mathbf{P}^{1}, \mathcal{O}_{\mathbf{P}^{1}}(i)\right)$. So we just have to show that $-K_{\tilde{\mathcal{B}}}$ restricts to $\mathcal{O}_{\mathbf{P}^{1}}(1)$ on the fibers of the projective bundle $E=\mathbf{P}\left(\mathcal{O} \oplus N_{S}\right)$. But this follows immediately from $(2.11)$ as $\rho^{*}\left(K_{\mathcal{B}}\right)$ has trivial restriction to the fibers while $\mathcal{O}_{\tilde{\mathcal{B}}}(E)$ restricts to $\mathcal{O}_{\mathbf{P}^{1}}(-1)$.

Physically the reason we are interested in this degeneration is because we may have singular fibers corresponding to an enhanced gauge symmetry along $S$, and we want to capture this geometry in a local model. So we now specialize $(2.9)$ to one of the Tate forms $[16,17]$ which imply that we have enhanced gauge symmetry along $S$. We investigate when we can follow this singularity through the degeneration to the normal cone and preserve the singularity type when we get to $B_{0}$, while "pushing the singularity away from $B_{3}$ " by putting the singularity at $S_{0} \subset E$, which is disjoint from $\tilde{B} \simeq B_{3}$. For simplicity, we stick to simply laced cases where we do not need to worry about monodromy issues. So we simply need to control the order of vanishing of the $a_{i}$ along $S$ and of the $\tilde{a}_{i}(t)$ along $S_{0}$. Let's say that the desired order of vanishing of $a_{i}$ along $S$ is $n_{i}$ for the appropriate gauge group. We need to assume the hypothesis that $n_{i} \leq i$. The ADE types of such singularities correspond to subgroups of $E_{8}$.

Claim: Suppose that the $a_{i}(t)$ satisfy the Calabi-Yau condition that it vanishes to order at least $i$ on $S \times\{0\}$, and furthermore that the $a_{i}(t)$ vanish to at least order $n_{i}$ on all of $S \times \mathbf{C}$. Then after the blowup which realizes the normal cone degeneration, the order of vanishing of $\tilde{a}_{i}(t)$ along $S_{0} \subset E \subset B_{0}$ is also at least $n_{i}$. 
Proof: By our assumptions, we can write $a_{i}(t)=s^{n_{i}} f_{i}(t)$ where $f_{i}(t)$ is holomorphic. Then $f_{i}(t)$ vanishes to order at least $i-n_{i}$ along $E$ after pulling back to $\tilde{\mathcal{B}}$. Since we only need the order of vanishing of $\tilde{a}_{i}(t)$ along $S_{0}$, we can compute in local coordinates near $S_{0}$. We have seen that $S_{0} \subset E$ is defined by $v=0$, hence $v / u=s / t$ is an affine $N_{S}$-valued fiber coordinate on the projective bundle $E=\mathbf{P}\left(\mathcal{O} \oplus N_{S}\right)$, vanishing along $S_{0}$. In this coordinate patch, $E$ is defined by $t=0$. It follow that in this patch $f_{i}(t)$ is divisible by $t^{i-n_{i}}$ and also that we may write $\tilde{a}_{i}(t)=a_{i}(t) / t^{i}$. Then we compute that

$$
\tilde{a}_{i}(t)=\frac{a_{i}(t)}{t^{i}}=\left(\frac{s}{t}\right)^{n_{i}}\left(\frac{f_{i}(t)}{t^{i-n_{i}}}\right)
$$

with $f_{i}(t) / t^{i-n_{i}}$ holomorphic. It follows that $\tilde{a}_{i}(t)$ vanishes to order at least $n_{i}$ along $S_{0}$ as claimed.

Example. We can achieve an $E_{8}$ gauge symmetry by requiring the order of vanishing of $\left(a_{1}, a_{2}, a_{3}, a_{4}, a_{6}\right)$ to be at least $(1,2,3,4,5)$. If we can choose sufficiently generic sections $a_{i}^{\prime} \in H^{0}\left(B,-i\left(K_{B}+D\right)\right)$ for $i=1,2,3,4$, and $a_{6}^{\prime} \in$ $H^{0}\left(B,-6 K_{B}-5 D\right)$, then the elliptic fibration

$$
y^{2}+a_{1}^{\prime} s y+a_{3}^{\prime} s^{3}=x^{3}+a_{2}^{\prime} s^{2} x^{2}+a_{4}^{\prime} s^{4} x+a_{6}^{\prime} s^{5}
$$

over $B_{3}$ has an $E_{8}$ singularity along $S$.

For $i=1, \ldots, 4$, it is automatic that $a_{i}(t)=a_{i}^{\prime}(t) s^{i}$ vanishes to order at least $i$ along $S \times\{0\}$. But for $i=6, a_{6}^{\prime}(t) s^{5}$ need not vanish to order 6 along $s=t=0$ as required to get a Calabi-Yau. One way to achieve the desired vanishing is to replace $a_{6}^{\prime}(t) s^{5}$ with $a_{6}^{\prime}(t) s^{5} t$. Thus the desired degeneration is given by

$$
y^{2}+a_{1}^{\prime}(t) s y+a_{3}^{\prime}(t) s^{3}=x^{3}+a_{2}^{\prime}(t) s^{2} x^{2}+a_{4}^{\prime}(t) s^{4} x+a_{6}^{\prime}(t) s^{5} t
$$

To obtain the elliptic fibration $W_{2}$ over $\tilde{B}$, we divide $a_{i}(t)$ by $s^{i}$ and put $t / s=0$ to obtain

$$
y^{2}+a_{1}^{\prime} y+a_{3}^{\prime}=x^{3}+a_{2}^{\prime} x^{2}+a_{4}^{\prime} x
$$

where we have put $a_{i}^{\prime}=a_{i}^{\prime}(0)$. 
To obtain the elliptic fibration $W_{1}$ over $E$, we instead divide each $a_{i}^{\prime}(t)$ by $t^{i}$, introduce the local equation $\tilde{s}=s / t$ which vanishes along $S_{0}$, and set $t=0$. That gives

$$
y^{2}+\left.a_{1}^{\prime}\right|_{S} \tilde{s} y+\left.a_{3}^{\prime}\right|_{S} \tilde{s}^{3}=x^{3}+\left.a_{2}^{\prime}\right|_{S} \tilde{s}^{2} x^{2}+\left.a_{4}^{\prime}\right|_{S} \tilde{s}^{4} x+\left.a_{6}^{\prime}\right|_{S} \tilde{s}^{5}
$$

which has the required $E_{8}$ along $S_{0}$.

Another example is discussed in section 3.5.

\subsection{Caveats}

Our construction made use of the Tate form of the Weierstrass fibration. As was recently investigated in [18], it is not clear if every elliptic fibration with, say, $S U(5)$ singularities along a divisor $S$ can be put in this form. Although no counterexample was found, we see no a priori reason why it should be the case that every elliptic fibration with $S U(5)$ singularities along $S$ can be put in Tate form.

For the purpose of constructing global models along the lines of [2], the more relevant question however is not whether one can put the elliptic fibration in Tate form, but whether there exists a degeneration to a local model. If the elliptic fibration can be put in Tate form, then as we saw above it is fairly straightforward to construct such a degeneration. It is not clear to us how to construct such a degeneration directly from the Weierstrass form, as the conditions for having suitable singularities along $S$ are rather non-linear in this formulation. But this does not mean that such a degeneration does not exist. Indeed, in the next section we will see another approach to constructing global Calabi-Yau manifolds, where the existence of a degeneration is built in from the start. This method makes no reference to the Tate form, only to the Weierstrass form.

On the other hand, it clearly should not be true that every single elliptic fibration with $S U(5)$ singularities along $S$ admits such a degeneration. Let us suppose that along a sublocus of $S$, the $S U(5)$ singularity degenerates to a type of singularity that cannot be fit in $E_{8}$, like say $S U(100)$. Then plausibly the degeneration to a local $d P_{9}$ model should not exist, or should develop very bad singularities. 
As a rule of thumb, it seems reasonable to expect that the degeneration to the local $d P_{9}$ model exists if the singularities can be fit in $E_{8}$, as we saw explicitly in our construction above through the condition $n_{i} \leq i$. Fortunately this appears to be sufficient for phenomenological applications. For example for $S U(5)_{G U T}$ models, generically the worst we expect is singularities of type $E_{6}, S O(12)$ and $S U(7)$ on $S$, all of which can be fit in $E_{8} \cdot{ }^{2}$ It is interesting to contrast this with an alternative weak coupling limit, namely the Sen limit. Note that $E_{6}$ cannot be fit in any $S O(n)$, so we expect the Sen limit to be problematic, as was indeed found in [2]. For related recent work, see [19].

At any rate, the global-to-local degeneration gives a clearer justification for the use of Higgs bundle and spectral cover technology, since a $d P_{9}$-fibration with G-flux is equivalent to spectral data. Similarly it gives a better justification for the 'heterotic' approach to instanton effects in F-theory [20]. In the remainder, we would like to place this in the general context of weak coupling limits and the cutting-and-gluing approach.

\section{Gluing a Calabi-Yau from local pieces}

We have motivated the issue of degeneration on phenomenological grounds, and from the point of view of string dualities and weak coupling limits. However there is another good motivation, based on an analogy with topological field theories.

\subsection{Some aspects of topological field theories}

Let us give some motivation by first considering topological field theories [21]. We will not give a systematic exposition, but rather discuss a few general features that will be useful below.

A topological field theory on a closed manifold $M$ is a quantum field theory

\footnotetext{
${ }^{2}$ We are referring here to the singularity type of the fiber over a point in $S$, not the singularity type of the total space. It is the former that is relevant for the spectral cover/gauge theory approach.
} 
in which the correlation functions are independent of the background metric. Our main interest here is in the gluing axiom. If the space $M$ has a boundary $\partial M=\Sigma$, then the field theory produces a state in a Hilbert space $\mathcal{H}_{\Sigma}$ given by quantizing the fields on the boundary. Now we can take two manifolds $M_{1}$ and $M_{2}$ with boundary with boundary $\Sigma$, and glue them together. The gluing axiom states how the the correlation functions are obtained from $M_{1}$ and $M_{2}$. For the partition function, this takes the form

$$
Z_{M}=\sum_{v_{i} \in \mathcal{H}_{\Sigma}} Z_{M_{1}}\left(v_{i}\right) Z_{M_{2}}\left(v^{i}\right)
$$

where $v_{i}$ is a basis of $\mathcal{H}_{\Sigma}$ and $v^{i}$ is the dual basis of $\mathcal{H}_{\Sigma}^{*}$.

The partition function of a topological field theory on a manifold $M$ can thus be computed by cutting the manifold into pieces and applying the rule above. For each piece, the field theory determines a distinguished state in the tensor product of Hilbert spaces associated to the boundaries. If the pieces are simple enough, then we may be able to write down this state explicitly. Then we glue the pieces back together to obtain the partition function on $M$. For beautiful examples of this paradigm, see eg. [22] or [23].

For the discussion below, it will also be useful to recall some aspects of ChernSimons theory on a real $3 d$ manifold $M_{3}$, which we take to be closed for now. The way the Chern-Simons action is usually defined in the literature is as follows: we pick a real $4 d$ manifold $M_{4}$ such that $M_{3}=\partial M_{4}$, and we extend the gauge field $A$ over $M_{4}$. Then the action is defined as

$$
S_{C S}(A)=\frac{k}{8 \pi^{2}} \int_{M_{4}} \operatorname{Tr}\left(F_{A} \wedge F_{A}\right)
$$

One may show that this does not depend on extension that one picks, up to an integral ambiguity. Namely if one chooses a different extension, then by gluing the two choices into a closed manifold and evaluating the above action, we see that the difference between the two choices must be an integer, so that $e^{i S_{C S}}$ is well defined.

The extension is not always possible, and a more complete answer has been given in [24]. In fact it will be useful to consider the definition of the Chern- 
Simons-type invariants on a general manifold $X$, with dimension possibly greater than three. A bundle on $X$ can be thought of as an equivalence class of maps from $\gamma: X \rightarrow B G$, where $B G$ is the classifying space of the gauge group $G$. The bundle on $X$ is then identified with the pull-back of the universal bundle $E G$ over $B G$. We will take $G$ to be connected and simply connected, as this simplifies things a little bit.

Since characteristic classes behave naturally under pull-back, we can now consider the analogous problem for the image of the three-cycle $M$ under the map $\gamma$. Namely we ask for a bounding four-chain for $\gamma(M)$. For $G$ connected and simply connected, we have $H_{3}(B G, \mathbf{Z})=0$, so the homology class $\gamma_{*}[M]=0$ and such a bounding four-chain exists, which then comes equipped with a $G$ bundle. We then extend the connection and integrate $\frac{1}{8 \pi^{2}} \operatorname{Tr}(F \wedge F)$ as before. For higher dimensional $X$, the Chern-Simons action is not canonically defined if the first Pontryagin class does not vanish on $X$, and we subtract a contribution $\frac{1}{8 \pi^{2}} \operatorname{Tr}\left(F_{0} \wedge F_{0}\right)$ of a reference connection $A_{0}$.

For general $G$, we have to worry about issues resulting from torsion in the cohomology of $B G$, so we can only bound an integer multiple $n \gamma$ of $\gamma$. We can integrate over a bounding chain as before and then divide by $n$, but the result will only be well-defined up to $1 / n$ times an integer. To properly define the theory, have to fix a cohomology class in $H^{4}(B G, \mathbf{Z})$ which can be used to fix the ambiguity. When $G$ is connected and simply connected, we have $H^{4}(B G, \mathbf{Z})=\mathbf{Z}$ and we can just use the first Pontryagin class of the universal bundle.

The choice of extension is not unique, and by changing the choices, we again get a natural discrete ambiguity. When $X$ is a three-manifold, this gives an integer ambiguity. For more general $X$, we get such an integer ambiguity for every three-cycle, in other words the Chern-Simons form is defined only modulo the lattice $H_{3}(X, \mathbf{Z})$.

When $X$ has a boundary, then we can proceed similarly. We glue on another manifold with boundary to get a closed manifold, extend the gauge field, and then apply the discussion above. The result depends on a choice of extension, which introduces an ambiguity, and is not quite gauge invariant. Under a gauge 
transformation which is non-trivial on the boundary, we have

$$
S(A) \rightarrow S(A)+\frac{k}{8 \pi^{2}} \int_{\partial M} \operatorname{Tr}\left(A \wedge d g g^{-1}\right)-\frac{k}{24 \pi^{2}} \int_{M} \operatorname{Tr}\left(g^{-1} d g\right)^{3}
$$

where we can see the WZW term emerging. For simplicity, we assumed here that the bundle is trivial. In fact, Chern-Simons theory on $D \times \mathbf{R}$ (with $D$ a disk) is equivalent to a chiral sector of a WZW model on the boundary $S^{1} \times \mathbf{R}$ [25].

If the boundary is empty, then we see from the above expression that the Chern-Simons action shifts under a topological quantity, the degree of the map $g: M \rightarrow G$. This gives another interpretation of the ambiguities: they arise from large gauge transformations that are not continuously connected to the identity.

\subsection{Analogy between topological and holomorphic field theories}

In holomorphic field theories, there is obviously some dependence on the metric on $M$. Furthermore we cannot simply cut up a manifold without breaking holomorphy, so the conventional notion of a boundary and the gluing axiom is not so useful. Nevertheless there is an analogue of the cutting and gluing procedure in topological field theories which is compatible with holomorphy. It is given by the degeneration of the complex manifold to a normal crossing variety, like the degeneration we discussed in the last section.

For physicists, this is familiar for example from the gluing construction of conformal blocks in $2 d$ CFT. We can degenerate the Riemann surface into pieces joined by a long neck. The neck is described algebraically by the equation

$$
x y=q
$$

In the limit $q \rightarrow 0$, the Riemann surface degenerates to a union $\Sigma_{1} \cup_{p} \Sigma_{2}$ glued along a puncture $p$. Instead of (3.1) above, for small $q$ we can construct conformal blocks using a $q$-expansion of the form

$$
Z_{\Sigma}(R, q)=\sum_{e_{i} \in R} Z_{\Sigma_{1}}\left(e_{i}\right) q^{w\left(e_{i}\right)} Z_{\Sigma_{2}}\left(e^{i}\right)
$$


We want to do something similar with higher dimensional varieties. A suitable higher dimensional analogue of (3.4) is the normal crossing singularity.

A further intriguing aspect of such a picture is the interplay between the theory in the bulk and the theory on the boundary. In fact even though the theories we are dealing with are not topological, in a number of known examples we can express the theory at small coupling entirely on the boundary. For $F$ theory the previously known degeneration limits are the $S O(32)$ and $E_{8} \times E_{8}$ heterotic limits. In these limits, the Calabi-Yau splits into two pieces, and the heterotic theories may be thought of as living on the normal crossing divisor $[26,27,10]$, which we interpret as the boundary. In $[20,7]$, the Sen limit is discussed from the same point of view and gives a further example: the boundary is the IIB space-time. The superpotential can be computed either on the fourfold or in the boundary theory.

We would like to argue that this should hold more generally. Instead of the algebraic arguments that have been used for the heterotic and IIB limits, we want to discuss an argument that is more in the spirit of topological field theory. The holomorphic analogue of real and orientable $n$-dimensional manifold with boundary is an $n$-complex dimensional log Calabi-Yau manifold. The holomorphic analogue of the orientation of a real manifold is holomorphic $(n, 0)$-form on the log Calabi-Yau, and the holomorphic analogue of the boundary is the polar divisor of the $(n, 0)$-form, i.e. the log divisor [28, 29]. The statement below was already given fifteen years ago [30]. We will give a slight variation of the original argument.

The analogue of Chern-Simons theory on $M_{3}$ is holomorphic Chern-Simons theory on a Calabi-Yau three-fold $X_{3}$. We will proceed as above for conventional Chern-Simons theory: we try to extend our gauge fields on $X_{3}$ to a log Calabi-Yau fourfold $\left(X_{4}, X_{3}\right)$, whose boundary is $X_{3}$. Then we try to define the holomorphic Chern-Simons action as

$$
S_{C S}(A)=\frac{1}{8 \pi^{2}} \int_{X_{4}} \Omega^{4,0} \wedge \operatorname{Tr}\left(F_{A} \wedge F_{A}\right)
$$

This action would normally seems to depend on the extension one picks. The choice of extension may not be unique, and as before this gives rise to a natural 
ambiguity. Furthermore, the holomorphic Chern-Simons action is in general not canonically defined, but depends on a choice of base point. We can eliminate the dependence on a reference connection only when $\left.p_{1}\right|_{X_{3}}$ vanishes. If $p_{1}$ is nonvanishing, then we cannot assign the same value of the Chern-Simons action to every three-cycle in the same homology class. (This is very similar to the Picard group: if $c_{1}(L)$ does not vanish, then we do not get a canonical map from the line bundle $L$ to the Jacobian. Instead we get a torsor structure, and the combined data defines a differential character or Deligne cohomology class). However when tadpole cancellation is satisfied, eg. $p_{1}\left(V_{1}\right)+p_{1}\left(V_{2}\right)-p_{1}\left(T X_{3}\right)=0$ in the heterotic string, then the sum of the Chern-Simons contributions is defined. If one needs 5branes wrapped on curves to satisfy tadpole cancellation, then their contribution to the superpotential also has to be included.

We now use the fact that the logarithmic cohomology $H_{\log }^{k}\left(X_{4}\right)$ for our pair $\left(X_{4}, X_{3}\right)$ is isomorphic to $H^{k}(U)$, where $U=X_{4} \backslash X_{3}$. Further we have that $H^{2 n-k}\left(X_{4}, X_{3}\right) \cong H_{c}^{2 n-k}(U)$, where $H_{c}^{*}$ denotes compactly supported cohomology. It follows that we have a Poincaré duality map $H_{\log }^{k}\left(X_{4}\right) \rightarrow H_{2 n-k}\left(X_{4}, X_{3}\right)$. From the short exact sequence $0 \rightarrow \Omega_{X_{4}}^{p} \rightarrow \Omega_{X_{4}}^{p}\left(\log X_{3}\right) \rightarrow \Omega_{X_{3}}^{p-1} \rightarrow 0$ we get the following long exact sequence for $H_{\log }^{k}\left(X_{4}\right)$ :

$$
\ldots \rightarrow H^{k-2}\left(X_{3}\right) \rightarrow H^{k}\left(X_{4}\right) \rightarrow H_{\log }^{k}\left(X_{4}\right) \stackrel{\text { res }}{\rightarrow} H^{k-1}\left(X_{3}\right) \rightarrow \ldots
$$

This is precisely the Poincaré dual to the long exact sequence for relative homology

$$
\ldots \rightarrow H_{2 n-k}\left(X_{3}\right) \rightarrow H_{2 n-k}\left(X_{4}\right) \rightarrow H_{2 n-k}\left(X_{4}, X_{3}\right) \stackrel{\partial}{\rightarrow} H_{2 n-k-1}\left(X_{3}\right) \rightarrow \ldots
$$

Now let us take a class $\alpha=p_{1}(A)-p_{1}\left(A_{0}\right)$ in $H^{4}\left(X_{4}\right)$ which restricts to a trivial class in $H^{4}\left(X_{3}\right)$. From the long exact sequence

$$
\ldots \rightarrow H^{4}\left(X_{4}, X_{3}\right) \rightarrow H^{4}\left(X_{4}\right) \rightarrow H^{4}\left(X_{3}\right) \rightarrow \ldots
$$

we see that $\alpha$ lifts to $H^{4}\left(X_{4}, X_{3}\right) \cong H_{c}^{4}(U)$. So now we compute:

$$
\int_{X_{4}} \Omega \wedge\left(p_{1}(A)-p_{1}\left(A_{0}\right)\right)=\int_{D} p_{1}(A)-p_{1}\left(A_{0}\right)
$$




$$
\begin{aligned}
& =\int_{\partial D} \omega_{C S}(A)-\omega_{C S}\left(A_{0}\right) \\
& =2 \pi i \int_{X_{3}} \operatorname{res}(\Omega) \wedge\left(\omega_{C S}(A)-\omega_{C S}\left(A_{0}\right)\right)
\end{aligned}
$$

where $D \in H_{4}\left(X_{4}, X_{3}\right)$ is the Poincaré dual of $\Omega$. This is what we wanted to show.

Note that on a $\log$ Calabi-Yau, we can rewrite $\int_{X_{4}} \Omega^{4,0} \wedge \alpha$ as an expression on $X_{3}$ for any $\alpha \in H^{4}\left(X_{4}\right)$, whether or not the restriction to $H^{4}\left(X_{3}\right)$ is trivial. To see this, we note that the $(0,4)$ part of $\alpha$ is Dolbeault exact, so with $\alpha^{0,4}=\bar{\partial} \omega$ we can write

$$
\int_{U} \Omega^{4,0} \wedge \bar{\partial} \omega=\left.2 \pi i \int_{X_{3}} \Omega^{3,0} \wedge \omega\right|_{X_{3}}
$$

where we used the Stokes and Cauchy theorems. However to relate this to the Chern-Simons action, we need the assumption on the first Pontryagin class.

In general it may not be possible to extend the gauge field $A$ in this way, but we can still fall back to the definition of the Chern-Simons form in [24], which we reviewed above. However we see that there's a further issue. The superpotential in $4 d N=1$ supergravity is a section of a line bundle $\mathcal{L}$ over the moduli space, so there is no room for periodic identifications. Also, the ambiguities of the holomorphic Chern-Simons action correspond to the periods of $\Omega^{3,0}$, which are usually dense in $\mathbf{C}$ (for example if the rank of $H_{3}\left(X_{3}, \mathbf{Z}\right)$ is at least three). This is not a problem if one is only interested in the derivatives, but it seems problematic whenever we are interested in the value of the action.

This issue reappears in superpotentials for brane configurations more generally. For example the superpotential for a brane wrapped on a two-cycle $C$ in a Calabi-Yau three-fold is given by [31]

$$
W=\int_{\Gamma} \Omega^{3,0}
$$

where $\partial \Gamma=C-C_{0}$ and $C_{0}$ is a holomorphic reference curve in the same homology class. This superpotential is actually closely related to the holomorphic ChernSimons action $[28,30,32,33]$. For holomorphic bundles, the Chern-Simons action is independent of the choice of hermitian metric and can be written as (3.12) where 
$C-C_{0}$ is the Chern character $\mathrm{ch}_{2}$, thought of as a Chow class [32]. As noted in [34], the description using $\mathrm{ch}_{2}$ is then naturally extended to coherent sheaves and even the derived category. By changing the choice of $\Gamma$, again we have an ambiguity by $H_{3}(X, \mathbf{Z})$, whose image under pairing with $\Omega^{3,0}$ is generally dense.

Nevertheless in string compactification this problem of periodic identifications is never an issue, as there are additional terms in the superpotential. ${ }^{3}$ This is another instance of the principle that the individual terms may be problematic, and only the sum of the terms has to be well-defined. For example in the heterotic string we have $W \sim \int_{X} \Omega^{3} \wedge\left(H+\omega_{C S}\right)$ with $d H+d \omega_{C S}=0$. Since $d H$ is not identically zero, $H$ does not live in $H^{3}(X, \mathbf{Z})$ but in a torsor, a principal fiber bundle with structure group $H^{3}(X, \mathbf{Z})$. However $H+\omega_{C S}$ does define a class in $H^{3}(X, \mathbf{Z})$, and $W$ is well-defined up to the usual ambiguity in picking $\Omega^{3,0}$. A similar phenomenon occurs in type IIB.

We believe that this perspective illuminates the role of Calabi-Yau fourfolds in the hierarchy of holomorphic field theories. The above expression (3.6) is of course very reminiscent of the flux superpotential that one encounters in $F$-theory. Indeed, it seems very natural to think about the flux superpotential in this way. Given an $F$-theory compactification with $G$-flux in a degeneration limit, let us consider the part of the flux which comes from $W_{1}$ or $W_{2}$ separately. We will briefly mention the other possible fluxes later.

Up to a shift in the quantization law, we may always express the cohomology class of the $G$-flux as $\mathrm{G} / 2 \pi=p_{1}(V)$ for some bundle $V$ on $Y$. According to [35] the shift is given by $-\frac{1}{2} p_{1}\left(T X_{4}\right)$ on a compact real eight-dimensional manifold. In our context we have instead a log Calabi-Yau manifold $\left(X_{4}, X_{3}\right)$. We propose that the tangent bundle $T X_{4}$ should be replaced by the logarithmic tangent bundle $\Theta \equiv T X_{4}\left(-\log X_{3}\right)$, which is the subbundle of $T X_{4}$ generated in coordinates by $\partial / \partial z_{1}, \partial / \partial z_{2}, \partial / \partial z_{3}$, and $z_{4} \partial / \partial z_{4}$, where $z_{4}=0$ is a local equation for $X_{3} \subset X_{4}$. If $X_{4}$ is a component of a normal crossings variety $Y_{0}=X_{4} \cup X_{4}^{\prime}$ whose components intersect along $Z=X_{3}$, then $T X_{4}\left(-\log X_{3}\right)$ is the restriction to $X_{4}$ of the logarithmic tangent bundle $T Y_{0}(-\log Z)$ introduced in section 2.1.

\footnotetext{
${ }^{3}$ We are grateful to E.Witten for illuminating comments in this regard.
} 
Thus we can write the superpotential as

$$
W=\frac{1}{2 \pi} \int_{X_{4}} \Omega^{4,0} \wedge \mathrm{G}=\int_{X_{4}} \Omega^{4,0} \wedge\left[p_{1}(V)-\frac{1}{2} p_{1}(\Theta)\right]
$$

Here we are implicitly assuming that $\left.\mathrm{G}\right|_{X_{3}}$ vanishes in $H^{4}\left(X_{3}\right)$, otherwise we should subtract the contribution of a reference connection as discussed above. Then as before we get:

$$
\begin{aligned}
W & =\int_{D}\left[p_{1}(V)-\frac{1}{2} p_{1}(\Theta)\right] \\
& =2 \pi i \int_{X_{3}} \Omega^{3,0} \wedge\left[\omega_{3}\left(\left.V\right|_{X_{3}}\right)-\frac{1}{2} \omega_{3}\left(T X_{3}\right)\right]
\end{aligned}
$$

with $\omega_{3}$ denoting the Chern-Simons form. Here we have used that $\left.p_{1}(\Theta)\right|_{X_{3}}=$ $p_{1}\left(T X_{3}\right)$. This follows from the exact sequence

$$
\left.0 \rightarrow T X_{3} \rightarrow T X_{4}\left(-\log X_{3}\right)\right|_{X_{3}} \rightarrow \mathcal{O}_{X_{3}} \rightarrow 0
$$

which is easily checked using local coordinates.

For the local $d P_{9}$-fibration discussed in the previous section, this ought to reproduce the holomorphic Chern-Simons theory on the boundary $Z$ with a single $E_{8}$ gauge group, if we restrict to allowed $F$-theory fluxes. ${ }^{4}$ This has previously been noted in [36]. We will show in section 4.3 using the cylinder mapping for $d P_{9}$-fibrations that at least we get the same critical set, corresponding to holomorphic $E_{8}$ bundles. But the point is really that this is a rather general property of periods on a log Calabi-Yau, and so quite generally we get such a Chern-Simons theory living on the boundary for each local piece of our global model.

Besides the fluxes above, there are additional G-fluxes on $W_{1} \cup_{Z} W_{2}$. They come either from $H^{3}(Z, \mathbf{Z})$ using the boundary map of the Mayer-Vietoris sequence, or from G-fluxes that have a pole along $Z$. This gives rise to extra terms

\footnotetext{
${ }^{4}$ It is natural to conjecture an analogous holographic relation for a $G_{2}$-manifold with superpotential $W=\int\left(\omega_{3}(A)+i \Phi\right) \wedge d\left(\omega_{3}(A)+i \Phi\right)$ and holomorphic Chern-Simons theory on its Calabi-Yau threefold boundary.
} 
in the superpotential on the boundary of the form $\int_{X_{3}} \Omega^{3,0} \wedge H$. We can understand this intuitively by dualizing the G-fluxes to homology classes, so that we can interpret the superpotential as a period integral. The G-fluxes considered above are cohomology classes on a local piece, so they dualize to a homology class on that local piece. The superpotential $\int_{X_{3}} \Omega^{3,0} \wedge H$ instead comes from periods of $\Omega^{4,0}$ over vanishing cycles, i.e. homology classes which disappear in the $t \rightarrow 0$ limit. In addition, there are typically also four-cycles which are not closed on the local pieces individually, and have to 'pass through the neck.' This is easily seen using the Mayer-Vietoris sequence. Periods over such four-cycles diverge with $\log (t)$ and give perturbative corrections to the superpotential on the boundary. Finally, all these periods receive corrections which are analytic in $t$, which we should interpret as instanton corrections on the boundary. We will discuss this in a more precise language in section 4.

From here it is a natural step to conjecture that in a degeneration limit, the full theory may be thought of as living on the boundary, not just the holomorphic sector for which we argued above. As evidence that this is indeed correct, we can again cite the heterotic and IIB limits of $F$-theory.

Let us make some side remarks. One can try to generalize many other known arguments used for topological field theories. A particularly interesting one is the 'holographic' relation between Chern-Simons theory on $M_{3}$ and WZW-models on $M_{2}=\partial M_{3}$, which should have an analogue in a relation between holomorphic Chern-Simons theory on a log Calabi-Yau three-fold $\left(X_{3}, X_{2}\right)$ and a $4 d$ version of WZW models on $X_{2}=\partial X_{3}$. Under a gauge transformation, we have

$$
S(A) \rightarrow S(A)+\frac{1}{8 \pi^{2}} \int_{\partial X_{3}} \Omega^{2,0} \wedge \operatorname{Tr}\left(A \wedge d g g^{-1}\right)-\frac{1}{24 \pi^{2}} \int_{X_{3}} \Omega^{3,0} \wedge \operatorname{Tr}\left(g^{-1} d g\right)^{3}
$$

where we see a holomorphic version of the WZW term emerging. Note that the last two terms only depend on the values of the fields at the boundary, and we have ambiguities corresponding to periods. This holomorphic WZW term was previously studied in [37].

It could also be interesting to consider Grassmann versions of these theories, which are used in the context of the twistor string approach to $N=4$ super YangMills theory [38]. The analogy with $F$-theory suggests that instanton corrections 
in the twistor string can be computed with Hodge theoretic methods by lifting to a higher dimensional space. One can see hints of this by thinking of D3-branes as obtained from compactifying $M 5$-branes on an elliptic curve. In the weak coupling limit, the elliptic curve becomes nodal and the D3-brane theory should be thought of as living on the nodal divisor. One would like a twistor version of this relation.

As a further side remark, another part of the analogy says that the analogue of correlators of Wilson loops in Chern-Simons theory are invariants associated to holomorphic curves in the holomorphic Chern-Simons theory [30, 29, 39]. In the abelian case, the holomorphic Wilson loop operator associated to a curve $C$ is defined as

$$
\mathcal{W}_{C}=\exp \int_{C} \Omega^{1,0} \wedge A^{0,1}
$$

where $\Omega^{1,0}$ is an orientation on $C$. In the non-abelian case, one would presumably define it as $\left.\operatorname{Det} \bar{\partial}_{A}\right|_{C}$. In the twistor context, it has been found that the amplitudes of $N=4$ Yang-Mills theory can be computed from such holomorphic Wilson loops [40]. This indicates an interesting kind of duality between instanton corrections in supertwistor space and holomorphic Wilson loops in a dual supertwistor space. It would be very interesting if some version of this duality exists more generally.

At any rate, we see that from the above point of view it is very natural to imagine constructing a Calabi-Yau manifold by gluing together local pieces, independent of any phenomenological considerations.

The Calabi-Yau obtained in this way will have normal crossing singularities. For many purposes, such a singular Calabi-Yau is practically as good as a smooth Calabi-Yau. The main difference is that instead of ordinary differential forms, we should consider logarithmic differential forms $\Omega^{\bullet}(\log D)$, which behave nicely under a smoothing. Nevertheless one would like to know if the normal crossing singularities can indeed be smoothed, for otherwise one might doubt that the theory exists for finite $t$. For applications to $F$-theory, one would also like to know if the Calabi-Yau obtained from smoothing the normal crossing singularities admits an elliptic fibration with a section. 


\subsection{Smoothing criteria of Kawamata/Namikawa}

Thus we now consider a Calabi-Yau $Y_{0}$ obtained from gluing together a collection of local models into a normal crossing variety. Then we would like to know if we can deform it to a smooth global model.

According to Kawamata and Namikawa [8], the normal crossing variety can be smoothed if it admits a log structure, and some further mild conditions. See e.g. [41, 42, 43] for many results about log geometry.

Kawamata and Namikawa consider the rather general set-up where the singular Calabi-Yau $d$-fold is of the form $\cup_{Z_{j}} W_{i}$. Then they prove that a smoothing exists if $Y_{0}$ is Kähler, admits a log structure, and has $H^{1}\left(Y_{0}\right)=H^{d-1}\left(\tilde{Y}_{0}\right)=0$, where $\tilde{Y}_{0}$ denotes the normalization of $Y_{0}$. Locally, $Y_{0}$ is isomorphic to a hypersurface $z_{1} \cdots z_{r}=0$ in a neighbourhood of the origin in $\mathbf{C}^{d+1}$, and the deformation corresponds to $z_{1} \cdots z_{r}=t$ locally.

For simplicity we will only consider the case $Y_{0}=W_{1} \cup_{Z} W_{2}$. Concretely, Kawamata and Namikawa prove that a smoothing of $W_{1} \cup_{Z} W_{2}$ exists in this case under the following conditions:

1. $Z \in\left|-K_{W_{i}}\right|$; in other words, each pair $\left(W_{i}, Z\right)$ is a log Calabi-Yau.

2. $H^{1}\left(\mathcal{O}_{W_{i}}\right)=H^{d-1}\left(\mathcal{O}_{W_{i}}\right)=0$. By a Mayer-Vietoris argument, this gives $H^{1}\left(Y_{0}\right)=0$, and clearly $H^{d-1}\left(\tilde{Y}_{0}\right)=0$ since $\tilde{Y}_{0}=W_{1} \coprod W_{2}$.

3. $Y_{0}$ should be Kähler. This is satisfied if there are ample divisors $H_{i}$ on $W_{i}$ such that $\left.H_{1}\right|_{Z}$ is linearly equivalent to $\left.H_{2}\right|_{Z}$; then we get an ample line bundle on $Y_{0}$ yielding a projective embedding, and thus it follows that $Y_{0}$ is Kähler.

4. $N_{Z / W_{1}} \otimes N_{Z / W_{2}}=\mathcal{O}_{Z}$. This is called the $d$-semi-stability condition [44], where $d$ is the dimension of $W_{i}$. By proposition 1.1 of [8], $d$-semi-stability is equivalent to the existence of a log structure.

Examples of smooth Calabi-Yau three-folds constructed by this method can be found for example in [8] and [45]. 
The proof of the theorem proceeds as follows. For each logarithmic structure, one can show that the obstruction to a deformation can be lifted order by order in perturbation theory. Then, the deformation is 'representable' over the ring of formal power series on the disk.

To show that one has an actual deformation, one proceeds as follows. ${ }^{5}$ In the analytic category, one knows that the Kuranishi space (i.e. the actual analytic deformation space) always exists, and then the formal power series result above shows that there is an analytic smoothing of the variety.

In the algebraic category one can make a similar argument, with the additional assumption that $H^{2}\left(\mathcal{O}_{Y_{0}}\right)=0$. If one starts with an ample line bundle on $Y_{0}$, then the assumption on $H^{2}$ above implies that the line bundle extends to the analytic smoothings. It follows that the family extends to a projective family by the existence of the Hilbert scheme. So again one can match the formal deformation with an actual deformation.

In order to apply the smoothing theorem of Kawamata/Namikawa to our situation, there are two issues that we should confront. The first is that Kawamata/Namikawa by itself does not guarantee the existence of a Weierstrass fibration. This will be addressed in the next subsection. A second problem is that the Kawamata/Namikawa theorem assumes the local pieces to be smooth. On the other hand, we are typically interested in the case that $W_{1}$ has interesting singularities, to get non-trivial non-abelian gauge groups. We may resolve the singularities and then apply Kawamata/Namikawa, but it is not completely clear that the exceptional cycles are preserved by the deformation, so that we can blow them back down.

Let us think about this problem from a physical perspective. The discriminant locus $\Delta$ of the elliptic fibration of $W_{1}$ decomposes into two pieces: a multiple of $S_{0}$ denoted by $\Delta_{0}$, and a remainder $\Delta^{\prime}$ which intersects $S_{0}$ in the matter curves. We want $\Delta_{0}$ to be preserved under the smoothing. In gauge theory language, this means that the gauge group should not get Higgsed under smoothing. When $S_{0}$ is a del Pezzo, there are no fields in the adjoint representation of the gauge group, so $\Delta_{0}$ won't deform by itself. However $\Delta_{0}$ might recombine with $\Delta^{\prime}$. In gauge

\footnotetext{
${ }^{5}$ We are grateful to Y.Kawamata for correspondence on this issue.
} 
theory language, this corresponds to Higgsing the gauge group by the charged matter located at the intersection of $\Delta^{\prime}$ with $S_{0}$.

So can this happen? Since we are only considering holomorphic questions here, we should ignore the $D$-terms. Consider a general superpotential $W$ in the charged fields $\phi, \tilde{\phi}$ on $\Delta_{0} \cap \Delta^{\prime}$, and consider $t$-dependent deformations. The problem comes from the $t$-derivative, if we have terms of the form

$$
W \supset P(t)+Q(t) \operatorname{Tr}(\phi \tilde{\phi})+\ldots
$$

which leads to $\phi \tilde{\phi} \sim P^{\prime}(t) / Q^{\prime}(t)$ for small $t$. However, substituting the VEVs in the derivatives of $W$ with respect to $\phi, \tilde{\phi}$, we would get a non-trivial equation for $t$, whereas we know by Kawamata/Namikawa that there is a one-parameter family parametrized by $t$. So perhaps $t$-dependent terms for $\operatorname{Tr}(\phi \tilde{\phi})$ are not generated, and it is consistent to set the charged fields to zero.

This argument is far from watertight. The question should be closely related to the choice of log structure. At any rate, we will see some examples later where we can do the deformation explicitly, and the gauge group along $S_{0}$ is preserved.

\subsection{Criteria for the existence of an elliptic fibration}

Let us assume that the central fiber $Y_{0}$ carries an elliptic fibration. In the main case studied in this paper the generic elliptic fibers are smooth, but this is not necessarily true for other interesting examples [7]. We really want the fibers $F$ to be connected and satisfy $\chi\left(F, \mathcal{O}_{F}\right)=0$. We would now like to see if the smoothing deformation provided by Kawamata and Namikawa preserves an elliptic fibration structure. This would be simplest if there were numerical criteria for the existence of an elliptic fibration on a variety. Such criteria are not known in complete generality. However there are criteria which cover most cases of interest, and which are valid in our situation, as was shown recently in [9].

Let us start with some general remarks. Suppose that a variety $X$ of dimension $d$ has an elliptic fibration $\pi: X \rightarrow B$. Consider an ample line bundle $L_{B}$ on $B$, and denote $L=\pi^{*} L_{B}$. Since $L_{B}$ is ample on $B$, for $m$ large enough the space of sections $H^{0}\left(X, L^{\otimes m}\right)$ will give a map from $X$ to projective space such that 
the image is isomorphic to $B$. This encapsulates an important idea in algebraic geometry, that rational maps often come from divisors in the manner above, and that we can try to characterize the morphism through the divisor $L$. In other words, instead of the data $(X, \pi)$ we will consider pairs $(X, L)$, from which $\pi$ can be reconstructed. The projection constructed from $H^{0}\left(X, L^{\otimes m}\right)$ with $m$ large enough is sometimes called the Iitaka fibration associated to $L$. The fibers of this limiting fibration are connected. Note that as necessary conditions for $L$ to be of the form $L=\pi^{*} L_{B}$, we have the following numerical criteria: $L \cdot C \geq 0$ for every curve $C$ on $X$ (i.e. $L$ is nef), $L^{d}=0$ and $L^{d-1} \neq 0$.

For the moment let's consider more general fibrations where the fibers $F$ are still curves, but not necessarily of (arithmetic) genus 1 . Let us consider the holomorphic Euler characteristic

$$
\chi\left(X, L^{m}\right)=\sum_{i}(-1)^{i} H^{i}\left(X, L^{m}\right)
$$

which is a deformation invariant. Using the Leray sequence for $\pi: X \rightarrow B$ one can show that

$$
\chi\left(X, L^{m}\right)=\chi\left(F, \mathcal{O}_{F}\right) \frac{m^{d-1}}{(d-1) !} L_{B}^{d-1}+\frac{m^{d-2}}{(d-2) !} L_{B}^{d-2} \cdot R+\mathcal{O}\left(m^{d-3}\right)
$$

where $R$ will be written more explicitly below. Thus $\chi$ typically grows as $m^{d-1}$. However, since $\chi\left(F, \mathcal{O}_{F}\right)=1-g, \chi$ will actually grow to order at most $m^{d-2}$ precisely when the fibers are elliptic. ${ }^{6}$

The divisor $R$ on $B$ is given by

$$
R=-\frac{1}{2} \chi\left(F, \mathcal{O}_{F}\right) K_{B}+\sum_{i}(-1)^{i} c_{1}\left(R^{i} \pi_{*} \mathcal{O}_{X}\right)
$$

\footnotetext{
${ }^{6} \mathrm{It}$ is interesting to note that by Riemann-Roch, the coefficient of $m^{d-1}$ in $\chi\left(X, L^{d}\right)$ is given by $(-1 / 2) L^{d-1} \cdot K_{X}$, so the coefficient of $m^{d-1}$ in (3.20) vanishes anyway on a Calabi-Yau. Indeed, by adjunction $c_{1}\left(K_{F}\right)=c_{1}\left(\left.K_{X}\right|_{F}\right)+c_{1}\left(N_{X / F}\right)$, but for any fibration we have $N_{X / F}$ is trivial. It follows that $(-1 / 2) K_{X} \cdot \pi^{*} L_{B}^{d-1}=(-1 / 2) \operatorname{deg}\left(\left.\left(K_{X}\right)\right|_{F}\right) L_{B}^{d-1}=\chi\left(F, \mathcal{O}_{F}\right) L_{B}^{d-1}$, since $\operatorname{deg}\left(\left.\left(K_{X}\right)\right|_{F}\right)=\operatorname{deg} K_{F}=2 g-2=-2 \chi\left(F, \mathcal{O}_{F}\right)$. So we conclude that the fibers must be elliptic when $K_{X} \sim 0$.
} 
Using that $\chi\left(F, \mathcal{O}_{F}\right)=0, R^{0} \pi_{*} \mathcal{O}_{X}=\mathcal{O}_{B}$, and $c_{1}\left(R^{1} \pi_{*} \mathcal{O}_{X}\right)=-\Delta_{X / B}$ is (minus) the class of the discriminant, we can simplify $\chi\left(X, L^{\otimes m}\right)$ to

$$
\chi\left(X, L^{\otimes m}\right)=\frac{m^{d-2}}{(d-2) !} L_{B}^{d-2} \cdot \Delta_{X / B}+\mathcal{O}\left(m^{d-3}\right)
$$

Therefore unless the discriminant locus is trivial, we see that the coefficient of $m^{d-2}$ must actually be non-zero. Using Riemann-Roch, one further shows that $L_{B}^{d-2} \cdot \Delta_{X / B}=L^{d-2} \cdot t d_{2}(X)$ as an expression on $X$, where $t d_{2}(X)$ is defined in terms of a resolution $h: \tilde{X} \rightarrow X$ as

$$
t d_{2}(X)=\frac{1}{12} h_{*}\left(c_{1}(\tilde{X})^{2}+c_{2}(\tilde{X})\right)
$$

So modulo some slight subtleties that we have glossed over, the idea is that we should have an elliptic fibration if we have a nef divisor $L$ on $X$, with the following properties: $H^{0}\left(X, L^{m}\right)$ should grow like $m^{d-1}$, so that the Iitaka fibration of $L$ gives a projection $\pi: X \rightarrow B$ with connected fibers where $B$ has dimension $d-1$. And $\chi\left(X, L^{m}\right)$ should grow like $m^{d-2}$, so that the fibers are elliptic. The latter is already a numerical criterion, and if $L^{d-2} \cdot t d_{2}(X)>0$ it turns out the former can be reformulated as one. Namely it is shown in [9] that a pair $(X, L)$ with $L^{d-2} \cdot t d_{2}(X)>0$ and $K_{X}$ nef is an elliptic fiber space if and only if

1. $L$ is nef, $L^{d}=0$ and $L^{d-1} \neq 0$ (the latter modulo torsion);

2. $L^{d-1} \cdot K_{X}=0$ and $L-\epsilon K_{X}$ is nef for $0 \leq \epsilon<<1$.

Of course the second condition is automatic if we have $K_{X} \sim 0$.

Now that we have a good characterization for the existence of an elliptic fibration, we can ask if it is preserved under a small deformation. It is worth keeping in mind that this fails in one of the simplest examples, namely the case of $K 3$ surfaces. The generic deformation of an elliptically fibered $K 3$ surface is certainly not elliptic. As another example, consider the product of two abelian varieties $A_{1} \times A_{2}$ and let $\pi$ be the projection on the second factor. The general deformation is a simple abelian variety which has no projection to a lower dimensional abelian variety. So there must be an extra condition if we are to expect an elliptic fibration after a deformation. 
The main problems are as follows. First, it is not guaranteed that $L$ deforms along with $X$. The obstruction lies in $H^{2}\left(X, \mathcal{O}_{X}\right)$, so if we require that $H^{2}\left(X, \mathcal{O}_{X}\right)$ vanishes then this problem is solved. ${ }^{7}$ This is the extra requirement that fails for $K 3$-surfaces and abelian varieties. Second, even when $L$ deforms, the condition that $L$ is nef is not an open condition in general. This problem is solved by an induction argument in [9], which can be made both in the algebraic and in the analytic category.

We conclude that if $Y_{0}$ admits an elliptic fibration with $\Delta_{X / B} \neq 0$ and $H^{2}\left(Y_{0}, \mathcal{O}_{Y_{0}}\right)=0$, then the deformation $Y_{t}$ provided by Kawamata and Namikawa is also elliptically fibered. As explained in the introduction, this gives a new and conceptually very interesting way to construct $F$-theory compactifications, by assembling the elliptic Calabi-Yau from more fundamental pieces. More generally, we see that $F$-theory is rather stable: when $H^{2}\left(X, \mathcal{O}_{X}\right)=0$, small complex structure deformations of $X$ preserve the existence of an elliptic fibration. This is why the moduli space of an $F$-theory compactification usually contains an open subset of the full complex structure moduli space of $X$.

\subsection{Example: bubbling off an $S U(5)_{G U T}$ model}

To keep things simple and illustrate the ideas, we consider the following example. We take $Y$ to be an elliptic fibration with section over $B_{3}=\mathbf{P}^{3}$, with $I_{5}$ singularities along a smooth irreducible divisor $S \subset B_{3}$ given by $z=0$. We explicitly degenerate this as

$$
Y_{t} \rightarrow W_{1} \cup_{Z} W_{2}
$$

where $W_{1}$ is a $d P_{9}$ fibration over $S$, and $W_{2}$ is an elliptic fibration over $\mathbf{P}^{3}$ with boundary $Z$, but different from $Y$. This is one of the simplest examples which does not have a $K 3$-fibration and therefore has no heterotic dual. See figure 2 for a schematic picture. It is clear that the same analysis may be applied to other examples, eg. the compact examples introduced in [2].

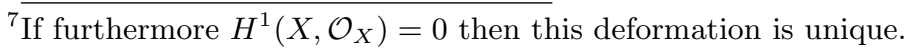


(A)

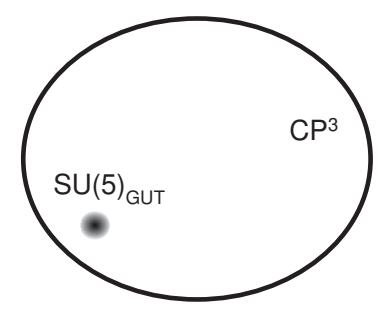

(B)

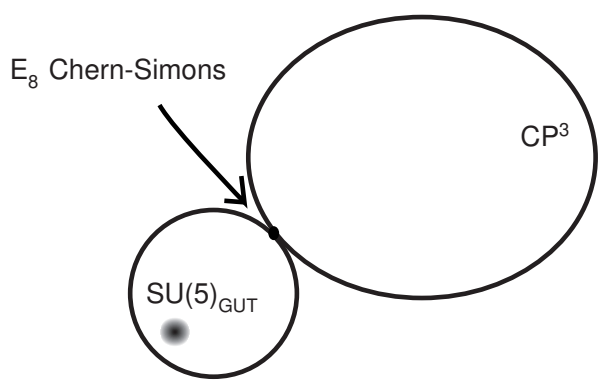

Figure 2: (A): Global model with $I_{5}$ singular fibers along a divisor $S$, corresponding to an $S U(5)_{G U T}$ gauge theory. (B): Degenerate version of the global model, in which the singular elliptic fibers describing the $S U(5)_{G U T}$ gauge theory have been pushed to a local $d P_{9}$-fibration.

The elliptic fibration over $\mathbf{P}^{3}$ can be written in the Tate form

$$
y^{2}+a_{1} x y+a_{3}=x^{3}+a_{2} x^{2}+a_{4} x+a_{6}
$$

where each of the $a_{i}$ are homogeneous polynomials of degree $4 i$ on $\mathbf{P}^{3}$. The $I_{5}$ condition along the hyperplane $z=0$ says that $a_{i}=b_{i} z^{i-1}$ for each $i$, where $b_{i}$ is a homogeneous polynomial of degree $3 i+1$ on $\mathbf{P}^{3}$. It will be convenient to write

$$
a_{i}=b_{i} z^{i-1}+c_{i} z^{i}
$$

There is some ambiguity in such a parametrization, as was the case in [18]. In the present example, we can make it more canonical by requiring $b_{i}$ to be independent of $z$.

Applying our results from Section 2.3, we can achieve the normal cone degeneration by simply inserting a factor of $t$ in front of each $b_{i}$ (since $i-n_{i}=1$ for all $i$ in the case of $I_{5}$ ). Doing so and changing the signs of $a_{1}$ and $a_{3}$ for convenience, we have the following elliptic fibration which extends to the normal 
cone degeneration:

$$
y^{2}=x^{3}+a_{1}(t) x y+a_{2}(t) z x^{2}+a_{3}(t) z^{2} y+a_{4}(t) z^{3} x+a_{6}(t) z^{5} .
$$

where

$$
a_{i}(t)=t b_{i} z^{i-1}+c_{i} z^{i}
$$

For $t \neq 0$, there is clearly an $I_{5}$ along $z=0$, and $a_{i}(t)$ vanishes to order $i$ along $z=t=0$ as required. Applying our previous results, at $t=0$ we get $W_{1}$ to be the local model with $I_{5}$ singularities.

Let's look at the limit in the neighborhood of the intersection of $W_{1}$ and $W_{2}$. In the notation of Section 2 we have the total space $\tilde{\mathcal{B}}$ of the normal cone degeneration, and for $t=0$ we have two components. In the current situation, one component (the base of $W_{2}$ ) is isomorphic to $\mathbf{P}^{3}$, and the other component (the base of $\left.W_{1}\right)$ is isomorphic to the projective bundle $\mathbf{P}(\mathcal{O} \oplus \mathcal{O}(1))$ over $\mathbf{P}^{2}$. Local coordinates near the intersection of these two components include $z$ and $(t / z)$, so that $t=0$ has two components because $t=z(t / z)$. The component $z=0$ is part of the exceptional divisor $E$ and $t / z=0$ gives $\mathbf{P}^{3}$.

To pass to the elliptic fibration over the normal cone degeneration, we replace the canonical bundle of $\mathbf{P}^{3}$ by the relative dualizing sheaf $\omega_{\pi}$. The effect of this is to require the removal of $i$ copies of the exceptional divisor from each $a_{i}(t)$. In our local coordinates, this means that we must replace each $a_{i}(t)$ by $\hat{a}_{i}=a_{i}(t) / z^{i}$, so that

$$
\hat{a_{i}}=\frac{t}{z} b_{i}+c_{i}
$$

Thus, (3.27) becomes after the normal cone degeneration

$$
\begin{aligned}
y^{2}=x^{3}+ & \frac{t}{z}\left(b_{1} x y+b_{2} x^{2}+b_{3} y+b_{4} x+b_{6}\right) \\
& +\left(c_{1} x y+c_{2} x^{2}+c_{3} y+c_{4} x+c_{6}\right)
\end{aligned}
$$

We restrict to the $\mathbf{P}^{3}$ component by putting $t / z=0$. This gives us $W_{2}$ :

$$
y^{2}=x^{3}+c_{1} x y+c_{2} x^{2}+c_{3} y+c_{4} x+c_{6} .
$$

The normal crossing divisor $Z=W_{1} \cap W_{2}$ is given by further setting $z=0$ in this equation. Note that the $c_{i}$ are sections of $\left(K_{B_{3}} \otimes \mathcal{O}(S)\right)^{-i}$, equivalently they 
are sections of powers of the $\log$ canonical bundle $K_{\left(B_{3}, S\right)}=K_{B_{3}} \otimes \mathcal{O}(S)$. So $\left.c_{i}\right|_{z=0}$ are sections of $K_{S}^{-i}$ by adjunction, and $Z$ is an elliptic Calabi-Yau threefold. Equation (3.31) is simply a generic elliptic fibration $W_{2}$ over $\mathbf{P}^{3}$ with the property that $\left(W_{2}, Z\right)$ is a $\log$ Calabi-Yau fourfold.

By setting $z=0$ in (3.30) and using $t / z$ as a local coordinate, we are describing a patch of $W_{1}$ near the infinity section, where it is glued onto $W_{2}$. The $I_{5}$ singularities are located at the zero section $S_{0}$, which is disjoint from the infinity section $S_{\infty}$. To see them explicitly, we rescale by $z / t$ and use $\tilde{z}=z / t$ as a local coordinate as we did in (2.21) where the more general $s$ was used instead of $z$. We get

$$
\begin{aligned}
y^{2}=x^{3}+ & b_{1} x y+b_{2} \tilde{z} x^{2}+b_{3} \tilde{z}^{2} y+b_{4} \tilde{z}^{3} x+b_{6} \tilde{z}^{5} \\
& +\left(c_{1} \tilde{z} x y+c_{2} \tilde{z}^{2} x^{2}+c_{3} \tilde{z}^{3} y+c_{4} \tilde{z}^{4} x+c_{6} \tilde{z}^{6}\right) .
\end{aligned}
$$

Here, all the $b_{i}$ and $c_{i}$ are evaluated at $z=0$. We recognize the typical form of a $d P_{9}$-fibration with $I_{5}$ singularities at $\tilde{z}=0$.

\subsection{The general case}

We return to the general case. Suppose conversely we are given two local models, i.e. two elliptically fibered $\log$ Calabi-Yau fourfolds $\left(W_{i}, Z\right)$ for $i=1,2$ which we glue along their common elliptically fibered boundary $Z$ to form $Y_{0}=$ $W_{1} \cup_{Z} W_{2}$. We want to know when this model corresponds to an F-theory limit, i.e. when $W_{1} \cup_{Z} W_{2}$ has a smoothing to an elliptically fibered Calabi-Yau fourfold $Y_{t}$.

As discussed earlier, we can find a smoothing when the Kawamata-Namikawa conditions hold. Let $B_{i}$ be the base of $W_{i}$ and let $S$ be the base of $Z$, so that $B_{0}=$ $B_{1} \cup B_{2}$ is the base of $Y_{0}$. We must assume that $H^{1}\left(\mathcal{O}_{W_{i}}\right)=0$ (for which it suffices to take $B_{i}$ with $H^{1}\left(\mathcal{O}_{B_{i}}\right)=0$ by the Leray spectral sequence), and the assumption that $Y_{0}$ is Kähler is easy to achieve in practice. The only other obstruction to the smoothability of $Y$ is the $d$-semistability condition $N_{Z, W_{1}} \otimes N_{Z, W_{2}} \simeq \mathcal{O}_{Z}$, which we now assume. With these assumptions, $Y_{0}$ smooths. If we further assume that the discriminant $\Delta_{Y_{0} / B_{0}}$ is non-empty and $H^{2}\left(\mathcal{O}_{Y_{0}}\right)=0$, then the smoothing is elliptically fibered and we are done. We will return to these general considerations 
later, but we digress slightly to specialize a bit.

As we have already discussed, the most interesting situation for $F$-theory occurs when one of the local models (say $W_{1}$ ) has a $d P_{9}$ fibration as well as a prescribed singularity along a divisor $S_{0} \subset B_{1}$ disjoint from $S$. We now claim that these assumptions imply that $B_{0}$ coincides with the limit $B_{0}$ of the degeneration to the normal cone of $S \subset B_{2}$, and we are back in the situation illustrated by examples in Section 2.3 and Section 3.5. Even still, we have gained something, as the more abstract Kawamata-Namakawa method makes it clear that our earlier assumptions of Tate form were not essential, so that the smoothing can be done if the prescribed singularities are given in a more general way. Furthermore, we can relax the hypotheses in the previous paragraph (for example by dropping the requirement of a heterotic dual) and achieve additional F-theory limits with more general bases $B_{0}$.

We now prove our claim. The assumption is that $B_{1}$ is fibered over $S$ by $\mathbf{P}^{1} \mathrm{~s}$, with disjoint sections $S$ and $S_{0}$. This implies that $B_{1}$ is the projectivization of a decomposable bundle $L_{1} \oplus L_{2}$, hence is isomorphic to $\mathbf{P}\left(\mathcal{O}_{S} \oplus L\right)$ for a line bundle $L$ on $S$, with either $L=L_{2} \otimes L_{1}^{-1}$ or $L=L_{1} \otimes L_{2}^{-1}$. Since $B_{1}-S_{0}$ is the total space of $N_{S / B_{1}}$, we see that $B_{1}$ must therefore be $\mathbf{P}\left(\mathcal{O}_{S} \oplus N_{S / B_{1}}\right)$. Next, since $N_{Z / Y_{i}}$ is the pullback of $N_{S / B_{i}}$ by the elliptic fibration, the $d$-semistabity condition is that the pullback of $N_{S / B_{1}} \otimes N_{S / B_{2}}$ is trivial. However, since $\operatorname{Pic}(S) \rightarrow \operatorname{Pic}(Z)$ is injective, we conclude that $N_{S / B_{1}} \simeq\left(N_{S / B_{2}}\right)^{-1}$. Then $B_{2}=\mathbf{P}\left(\mathcal{O}_{S} \oplus N_{S / B_{1}}\right) \simeq$ $\mathbf{P}\left(\mathcal{O}_{S} \oplus N_{S / B_{2}}^{-1}\right) \simeq \mathbf{P}\left(N_{S / B_{2}} \oplus \mathcal{O}_{S}\right)$. Note that $N_{S / B_{2}}$ is now identified with a neighborhood of $S_{0}$ and we have achieved the limit of the degeneration to the normal cone.

We now return to the general case and be more explicit, using Weierstrass form. We start with projective bases $B_{1}$ and $B_{2}$ with $H^{1}\left(B_{i}, \mathcal{O}_{B_{i}}\right)=0$ and $H^{2}\left(B_{i}, \mathcal{O}_{B_{i}}\right)=0$, sharing a common divisor $S$ with inverse normal bundles in the respective components. Let $K_{i}=K_{B_{i}}+S$ be the restriction of the dualizing sheaf of $B_{0}$ to $B_{i}$, and assume that $-4 K_{i}$ and $-6 K_{i}$ have enough sections $f_{i} \in$ $H^{0}\left(B_{i},-4 K_{i}\right)$ and $g_{i} \in H^{0}\left(B_{i},-6 K_{i}\right)$ with $\left.\left(f_{1}\right)\right|_{S}=\left.\left(f_{2}\right)\right|_{S}$ and $\left.\left(g_{1}\right)\right|_{S}=\left.\left(g_{2}\right)\right|_{S}$ so that we have a Weierstrass fibration

$$
y^{2}=x^{3}+f x+g,
$$


where $f$ and $g$ are the sections on $B_{0}$ obtained from the gluing.

Let's see what else we need for all the necessary conditions. The first condition was that the pairs $\left(W_{1}, Z\right)$ and $\left(W_{2}, Z\right)$ should be log Calabi-Yau spaces, but that is automatic since $f_{i}$ and $g_{i}$ were constructed from the log canonical bundles.

The remaining conditions are also easy to check or achieve. We have already checked that the $d$-semistability condition holds since it pulls back from the base. In order for $B_{0}$ to be projective, we need to find ample line bundles $L_{i}$ on $B_{i}$ which agree on $S$. This is easy to achieve if the restrictions of $\operatorname{Pic}\left(B_{i}\right)$ to $\operatorname{Pic}(S)$ are big enough; or we can just assume it. And there is nothing to check in the case of the normal cone degeneration since the degeneration can be achieved within projective geometry by blowups as we have seen. Thus, with the above mild assumptions, $Y_{0}$ deforms.

Finally, we want to what conditions are needed so that the deformation is elliptic. Since we are using Weierstrass form, we only need generic enough sections $f, g$ for the discriminant $\Delta=4 f^{3}+27 g^{2}$ to be a nontrivial divisor, so we assume that. Furthermore, $H^{2}\left(\mathcal{O}_{Y_{0}}\right)=0$ by a Leray spectral sequence argument.

Then we may apply the results of [9] discussed in Section 3.4, and $Y_{0}$ deforms to an elliptically fibered Calabi-Yau fourfold, with a built-in degeneration limit.

\section{The form of the Lagrangian in a degeneration limit}

As we explained, we are not really interested in complex spaces themselves, but in holomorphic field theories on such spaces. Such holomorpic field theories lead to interesting moduli spaces, and differential equations on bundles over these moduli spaces. Degeneration limits give rise to boundaries on such moduli spaces, and we now want to study how correlators (solutions of differential equations) behave near a boundary.

Let us think back for example about the case of conformal blocks of CFTs on a Riemann surface. The conformal blocks of a CFT are mathematically described by (twisted) $D$-modules, which are roughly speaking holomorphic vector bundles 
over the moduli space with a (projectively) flat connection. The connection usually develops a logarithmic singularity when the Riemann surface degenerates $\left(\nabla \sim d+L_{0} d t / t\right)$, and the monodromies give an action of the mapping class group on the correlators. This action plays an important role in two-dimensional CFT.

One expects similar phenomena for holomorphic field theories in higher dimensions, and $F$-theory provides another case study. In the large volume limit of $F$-theory the moduli space is a fibration, with base given by the complex structure moduli space of the Calabi-Yau and fiber given by the intermediate Jacobian $\mathcal{J}^{2}=H_{\mathbf{C}}^{3} /\left(F^{2} H_{\mathbf{C}}^{3}+H_{\mathbf{Z}}^{3}\right)$. In what follows we focus on the complex structure moduli and ignore the Jacobian. We get a $D$-module structure from the Gauss-Manin connection on the Hodge bundle over the complex structure moduli space of the Calabi-Yau.

In this section we want to understand the behaviour in a semi-stable degeneration limit. Fortunately the general aspects of this problem are well-understood. In appropriate coordinates, near the degeneration limit the Gauss-Manin connection takes the form

$$
\nabla=d+N \frac{d t}{t}
$$

where $N$ is a nilpotent matrix. Once we know the matrix $N$, we can use this to make qualitative statements about the behaviour of the effective lagrangian without having to calculate a single period integral. As we will see, the matrix $N$ can be deduced from the geometry of the central fiber. Thus this fits very well with the cutting-and-gluing approach.

\subsection{Asymptotics from monodromy}

On a smooth fourfold, the superpotential for the supergravity fields is the flux superpotential, which appears to take the form of a period integral:

$$
W=\frac{1}{2 \pi} \int_{Y} \Omega^{4,0} \wedge \mathrm{G}
$$

with $\mathrm{G} / 2 \pi-1 / 2 p_{1}(T Y)$ having integral periods. Thus we want to understand the behaviour of periods in a semi-stable degeneration limit, which is a classical 
problem in Hodge theory.

We have to make some caveats. For phenomenological applications we are not quite interested in smooth Calabi-Yau spaces, but in situations where certain exceptional cycles are contracted, in order to get non-abelian gauge symmetries. The above superpotential does not capture interactions of quantized $M 2$ solitons wrapped on exceptional cycles, since such degrees of freedom are much heavier and are not obtained by KK reduction from supergravity. Such degrees of freedom however become important in the limit that the exceptional cycles shrink to zero, and there exist branches of non-abelian solutions which are not captured by (4.2). Nevertheless we expect the $t$-dependent corrections to be qualitatively similar.

Further, the superpotential is often thought of as being defined on the infinite dimensional space of field configurations, i.e. it also depends on all the massive KK modes. Here we are only evaluating it on harmonic forms. Still let us see what we can learn from only a few general considerations.

The qualitative form of the periods in a degeneration limit is rather constrained, and can be understood using Hodge theory. We have given a brief review of the relevant material in appendix A. Here we will be interested in general properties of the case of a semi-stable degeneration $\pi_{\mathcal{Y}}: \mathcal{Y} \rightarrow \Delta$, where $Y_{t}$ is a smooth Calabi-Yau fourfold for $t \neq 0$, and $Y_{0}=W_{1} \cup_{Z} W_{2}$, with $\left(W_{1}, Z\right)$ and $\left(W_{2}, Z\right) \log$ Calabi-Yau spaces and $Z$ a Calabi-Yau threefold.

The lattice $H_{4}\left(Y_{t}, \mathbf{Z}\right)$ forms a local system over the $t$-plane away from $t=0$. We can use parallel transport to get an isomorphism with $H_{4}\left(Y_{t^{\prime}}, \mathbf{Z}\right)$ for $t^{\prime} \neq t$. Now let us circle around the origin of the $t$-plane. Then the cycles in $H_{4}\left(Y_{t}, \mathbf{Z}\right)$ get rearranged, and this is expressed as an automorphism $M$ of $H_{4}\left(Y_{t}, \mathbf{Z}\right)$ called the monodromy transformation. It is known in general that up to a base change (i.e. reparametrizing $t$ as $t \sim \tilde{t}^{k}$ ), the monodromy transformation is unipotent. In our situation with a semistable degeneration, the monodromy was already unipotent and so a base change is not necessary. We also introduce the logarithm $N=\log (M)$, which is nilpotent when $M$ is unipotent.

Let us collect the periods of $\Omega^{4,0}$ with respect to some basis of $H_{4}\left(Y_{t}, \mathbf{Z}\right)$ in a vector $\vec{\Pi}(t)$. Now the Schmid nilpotent orbit theorem says that as $t \rightarrow 0$ the 
periods are approximated in the following way:

$$
\vec{\Pi}(t) \sim e^{\frac{1}{2 \pi i} N \log (t)} \vec{\Pi}_{0}
$$

where $N$ is the logarithm of the monodromy matrix, and $\vec{\Pi}_{0}$ is independent of $t$. The expression on the right, i.e. the nilpotent orbit, may be thought of as a perturbative approximation to the periods. Thus we want to know the matrix $N$ and we want to know $\vec{\Pi}_{0}$. In other words, we want to know the limiting mixed Hodge structure associated to the degeneration $t \rightarrow 0$. In the following, we delve into a slightly lengthy analysis in order to derive this. Readers who might not want to follow this derivation may skip to equation (4.36), where we write the form of the superpotential that follows from our analysis.

\subsection{The limiting mixed Hodge structure}

As reviewed in appendix A, there are at least two ways for getting the limiting mixed Hodge structure. The approach we will use below is the Clemens-Schmid exact sequence, which allows us to compare the limiting mixed Hodge structure for $t \rightarrow 0$ with the mixed Hodge structure of the central fiber $Y_{0}$. An alternative approach, also briefly mentioned in appendix A, is to study the mixed Hodge structure of the logarithmic cohomology groups $H_{\log }^{k}\left(Y_{0}\right)$ of the central fiber.

The part of the Clemens-Schmid sequence that we will use is

$$
\cdots \rightarrow H_{6}\left(Y_{0}\right) \stackrel{\alpha}{\rightarrow} H^{4}\left(Y_{0}\right) \stackrel{i^{*}}{\rightarrow} H_{\lim }^{4}\left(Y_{t}\right) \stackrel{N}{\rightarrow} H_{\lim }^{4}\left(Y_{t}\right) \stackrel{\beta}{\rightarrow} H_{4}\left(Y_{0}\right) \rightarrow \cdots
$$

Since $Y_{0}$ is a deformation retract of $\mathcal{Y}$, we see that the homologies and comologies of $Y_{0}$ are identified with those of $\mathcal{Y}$. Then $i^{*}$ is identified with the usual pullback $i^{*}: H^{k}(\mathcal{Y}) \rightarrow H^{k}\left(Y_{t}\right)$. The maps $\alpha$ and $\beta$ are induced by Poincaré duality on $\mathcal{Y}$ and $Y_{t}$ respectively. All of the terms in the Clemens-Schmid sequence carry natural mixed Hodge structures. The maps $\alpha, i^{*}, N$, and $\beta$ are morphisms of mixed Hodge structures, shifting the degrees by $(5,5),(0,0),(-1,-1)$, and $(-4,-4)$ respectively.

Now we need the weight filtration for $H^{4}\left(Y_{0}\right)$. There is a general prescription which can be found in appendix A, but in the present case we can easily read it 
off from the Mayer-Vietoris sequence for $Y_{0}$, which is given by

$$
\begin{aligned}
\ldots & \rightarrow H^{3}\left(W_{1}\right) \oplus H^{3}\left(W_{2}\right) \stackrel{\mathrm{d}^{3}}{\rightarrow} H^{3}(Z) \rightarrow \\
\rightarrow H^{4}\left(Y_{0}\right) & \rightarrow H^{4}\left(W_{1}\right) \oplus H^{4}\left(W_{2}\right) \stackrel{\mathrm{d}^{4}}{\rightarrow} H^{4}(Z) \rightarrow \ldots
\end{aligned}
$$

This gives a short exact sequence

$$
0 \rightarrow \operatorname{coker}\left(\mathrm{d}^{3}\right) \rightarrow H^{4}\left(Y_{0}\right) \rightarrow \operatorname{ker}\left(\mathrm{d}^{4}\right) \rightarrow 0
$$

and so the weight filtration on $H^{4}\left(Y_{0}\right)$ is simply given by

$$
0 \subseteq \mathrm{W}_{3} \subseteq \mathrm{W}_{4}=H^{4}\left(Y_{0}\right)
$$

with $\operatorname{Gr}_{4} H^{4}\left(Y_{0}\right)=\operatorname{ker}\left(\mathrm{d}^{4}\right)$ and $\operatorname{Gr}_{3} H^{4}\left(Y_{0}\right)=\operatorname{coker}\left(\mathrm{d}^{3}\right)$.

The Clemens-Schmid sequence (4.4) induces exact sequences on the graded pieces. The Mayer-Vietoris sequence on homology is given by

$$
\ldots \rightarrow H_{k}\left(W_{1}\right) \oplus H_{k}\left(W_{2}\right) \rightarrow H_{k}\left(Y_{0}\right) \rightarrow H_{k-1}(Z) \rightarrow \ldots
$$

This shows that the non-zero graded pieces of $H_{6}\left(Y_{0}\right)$ are of weights -6 and -5 , while those of $H_{4}\left(Y_{0}\right)$ are of weights -4 and -3 .

Since $\mathrm{Gr}_{-7} H_{6}\left(Y_{0}\right)=0$ and $\mathrm{Gr}_{1} H^{4}\left(Y_{t}\right)=0$, the relevant graded piece of (4.4) gives an isomorphism

$$
\mathrm{Gr}_{3} H^{4}\left(Y_{0}\right) \cong \mathrm{Gr}_{3} H_{\lim }^{4}\left(Y_{t}\right)
$$

so that

$$
\mathrm{Gr}_{3} H_{\mathrm{lim}}^{4}\left(Y_{t}\right) \simeq \operatorname{coker}\left(\mathrm{d}^{3}\right)
$$

We also have the isomorphism

$$
\operatorname{Gr}_{5} H_{\mathrm{lim}}^{4}\left(Y_{t}\right) \stackrel{N}{\simeq} \operatorname{Gr}_{3} H_{\mathrm{lim}}^{4}\left(Y_{t}\right)
$$

by a general property of the monodromy weight filtration, or alternatively from another graded piece of (4.4). 
Let $p$ be the smallest integer such that $\operatorname{Gr}_{p} H^{4}\left(Y_{t}\right) \neq 0$. We claim $p=3$. If $p<3$, we have from (4.4) the short exact sequence

$$
\operatorname{Gr}_{p} H^{4}\left(Y_{0}\right) \rightarrow \operatorname{Gr}_{p} H^{4}\left(Y_{t}\right) \stackrel{N}{\rightarrow} \operatorname{Gr}_{p-2} H^{4}\left(Y_{t}\right)
$$

Since the left and right terms vanish by our assumptions, it follows that also $\mathrm{Gr}_{p} H^{4}\left(Y_{t}\right)=0$, a contradiction. Because $N^{k}: \mathrm{Gr}_{4+k} \rightarrow \mathrm{Gr}_{4-k}$ is an isomorphism, we see that the only non-zero graded pieces of $H^{4}\left(Y_{t}\right)$ are $\mathrm{Gr}_{3}, \mathrm{Gr}_{4}$ and $\mathrm{Gr}_{5}$, and the matrix $N$ satisfies $N^{2}=0$. Since the weight filtration is non-trivial, we also have $N \neq 0$. More generally this argument shows that if the central fiber only has strata up to codimension $k$, so that if $\mathrm{W}_{n-m} H^{n}\left(Y_{0}\right)=0$ for $m>k$, then we will have $N^{k+1}=0$.

To summarize, we found that $N^{2}=0$ but $N \neq 0$. The monodromy weight filtration on $H^{4}\left(Y_{t}\right)$ therefore has the form

$$
0 \subseteq \mathrm{W}_{3} \subseteq \mathrm{W}_{4} \subseteq \mathrm{W}_{5}=H_{\lim }^{4}\left(Y_{t}\right)
$$

where $\mathrm{W}_{3} H^{4}\left(Y_{t}\right)=\operatorname{im}(N)$ and $\mathrm{W}_{4} H^{4}\left(Y_{t}\right)=\operatorname{ker}(N)$. We also found the following isomorphisms of Hodge structures:

$$
\operatorname{Gr}_{5} H_{\lim }^{4}\left(Y_{t}\right) \stackrel{N}{\simeq} \operatorname{Gr}_{3} H_{\lim }^{4}\left(Y_{t}\right) \simeq \operatorname{Gr}_{3} H^{4}\left(Y_{0}\right) \simeq \operatorname{coker}\left(\mathrm{d}^{3}\right)
$$

One can say more than this. From (4.4) we also see that

$$
\operatorname{Gr}_{4} H_{\lim }^{4}\left(Y_{t}\right) \simeq \operatorname{Gr}_{4} H^{4}\left(Y_{0}\right) / \operatorname{ker}\left(i^{*}\right) \simeq \operatorname{ker}\left(\mathrm{d}^{4}\right) / \operatorname{ker}\left(i^{*}\right)
$$

as well as

$$
\mathrm{W}_{4} H_{\lim }^{4}\left(Y_{t}\right) \simeq \mathrm{W}_{4} H^{4}\left(Y_{0}\right) / \operatorname{ker}\left(i^{*}\right)=H^{4}\left(Y_{0}\right) / \operatorname{ker}\left(i^{*}\right)
$$

Now the map $\alpha$ consists of Poincaré duality $H_{6}\left(Y_{0}\right) \cong H_{6}(\mathcal{Y}) \rightarrow H^{4}(\mathcal{Y}, \partial \mathcal{Y})$, which is an isomorphism, followed by the natural map $H^{4}(\mathcal{Y}, \partial \mathcal{Y}) \rightarrow H^{4}(\mathcal{Y}) \cong H^{4}\left(Y_{0}\right)$. Furthermore, by the Thom isomorphism we have $H^{4}(\mathcal{Y}, \partial \mathcal{Y}) \cong H^{2}\left(Y_{0}\right)$. Then the 
geometric picture for $\operatorname{ker}\left(i^{*}\right)=\operatorname{im}(\alpha)$ is as follows. When we glue $W_{1}$ and $W_{2}$, we have to make certain identifications on the cohomology, as we can see from (4.5). Similarly we have to make certain identifications in homology. From (4.8) we see that when a four-cycle in $Z$ embeds non-trivially in $W_{1}$ and $W_{2}$, then it has to be identified in $Y_{0}$. Now the Poincare duals of these classes in $W_{1}$ and $W_{2}$ are not identified when we glue; they descend to two distinct cohomology classes in $Y_{0}$. But we expect that they should become equivalent when we deform to $Y_{t}$, since $Y_{t}$ is smooth and therefore has Poincaré duality. As a result a linear combination of these classes is in the kernel of $i^{*}$. We can see this a bit more easily using the logarithmic approach, using equation (A.24).

As we will see in a bit more detail in the next section, the remaining information in the flag structure (4.13) can also be extracted. We can now use this knowledge of the monodromy, together with the nilpotent orbit theorem, to compute the asymptotics of periods in the degeneration limit.

\subsection{Asymptotic form of the superpotential}

The nilpotent orbit theorem [14] says that the degeneration of Hodge structures behaves asymptotically like $\exp \left(\frac{1}{2 \pi i} \log (t) N\right) F_{\text {lim }}^{\bullet}$, where $F_{\text {lim }}^{\bullet}$ is the Hodge filtration of the limiting mixed Hodge structure on $H^{4}\left(Y_{t}\right)$. We recall this in the Appendix (cf. (A.26)). Here we apply it to compute the asymptotics of periods in our degeneration limit.

For the holomorphic four-form, we are interested in the $F^{4}$ component. Let $\Omega_{0} \in F^{4}$ be a nonzero element of the one-dimensional vector space $F^{4}$. Then $N \Omega_{0} \in F^{3} \cap \mathrm{W}_{3}$ is nonzero. This gives, since $N^{2}=0$

$$
\Omega(t) \sim \Omega_{0}(t) \equiv \Omega_{0}+\frac{1}{2 \pi i} \log (t) N \Omega_{0}
$$

In order to find the superpotential, we need to write the period map. Let us take a basis $\left\langle e^{i}, f^{j}, g^{k}\right\rangle$ for $\mathrm{W}_{5}=H^{4}\left(Y_{t}, \mathbf{Z}\right)$ which is adapted to the monodromy weight filtration. That is, the $e^{i}$ projects to a basis of $\mathrm{W}_{5} / \mathrm{W}_{4}$, the $f^{j}$ project to a basis $\mathrm{W}_{4} / \mathrm{W}_{3}$, and the $g^{k}$ span $\mathrm{W}_{3}$. The matrix $N$ acts as $N e^{i}=g^{i}, N f^{j}=N g^{k}=0$. We also consider the dual basis $\left\langle e_{i}, f_{j}, g_{k}\right\rangle$ for $H_{4}\left(Y_{t}, \mathbf{Z}\right)$. Then we can decompose 
$\Omega_{0}$ as

$$
\Omega_{0}=e^{i} \int_{e_{i}} \Omega_{0}+f^{j} \int_{f_{j}} \Omega_{0}+g^{k} \int_{g_{k}} \Omega_{0}
$$

and we can decompose the $F^{4}$ part of the nilpotent orbit as

$$
\Omega_{0}(t)=e^{i} \int_{e_{i}} \Omega_{0}+f^{j} \int_{f_{j}} \Omega_{0}+g^{k}\left(\frac{1}{2 \pi i} \log (t) \int_{e_{k}} \Omega_{0}+\int_{g_{k}} \Omega_{0}\right)
$$

Using the basis $\left\langle e^{i}, f^{j}, g^{k}\right\rangle$ to fix an isomorphism $\Pi: H^{4}\left(Y_{t}, \mathbf{Z}\right) \cong \mathbf{Z}^{\operatorname{dim} H^{4}}$, we can think of this as the period map. As our decomposition suggests, we can understand the properties of $\Omega_{0}$ in $F^{4} \cap \mathrm{W}_{5}=F^{4} H_{\text {lim }}^{4}\left(Y_{t}\right)$ by successively building up $\mathrm{W}_{5}$ from the pure Hodge structures on $\mathrm{Gr}_{5}, \mathrm{Gr}_{4}$ and $\mathrm{Gr}_{3}$ using the weight filtration.

First we consider the image of $\Omega_{0}$ in $F^{4} \mathrm{Gr}_{5}$, by which we mean the projection $\left(F^{4}+\mathrm{W}_{4}\right) / \mathrm{W}_{4}$. Here we can use the fact that $F^{4} \mathrm{Gr}_{5} \simeq F^{3} \operatorname{coker}\left(\mathrm{d}^{3}\right)$ is an isomorphism. Define $\left\langle d^{i}\right\rangle$ to be a basis for coker $\left(\mathrm{d}^{3}\right)$ which gets mapped to $\left\langle e^{i}\right\rangle$ and $\left\langle g^{i}\right\rangle$ under the isomorphisms $\operatorname{Gr}_{5} \cong \operatorname{Gr}_{3} \cong \operatorname{coker}\left(\mathrm{d}^{3}\right)$, and let $\left\langle d_{i}\right\rangle$ be its dual. Under the isomorphism we have

$$
\int_{e_{i}} \Omega_{0}=\int_{d_{i}} \Omega^{3,0}
$$

This is the expression we will use for the first term in (4.18).

In the next step, we try to lift this to $F^{4}\left(\mathrm{~W}_{5} / \mathrm{W}_{3}\right)=\left(F^{4}+\mathrm{W}_{3}\right) / \mathrm{W}_{3}$. Note that from the short exact sequence $0 \rightarrow \mathrm{W}_{4} \rightarrow \mathrm{W}_{5} \rightarrow \mathrm{Gr}_{5} \rightarrow 0$, it follows that $\mathrm{W}_{5} / \mathrm{W}_{3}$ sits in the following short exact sequence of mixed Hodge structures:

$$
0 \rightarrow \mathrm{Gr}_{4} \rightarrow \mathrm{W}_{5} / \mathrm{W}_{3} \rightarrow \mathrm{Gr}_{5} \rightarrow 0
$$

and we are interested in the $F^{4}$ part. We have already discussed $F^{4} \mathrm{Gr}_{5}$, and in fact we have $F^{4} \mathrm{Gr}_{4}=\left(F^{4} \cap \mathrm{W}_{4}+\mathrm{W}_{3}\right) / \mathrm{W}_{3}=0$. To see this, $\mathrm{Gr}_{4}$ has a weight four Hodge structure so could a priori have subspace of Hodge type (4,0). However, $\mathrm{Gr}_{4} H^{4}\left(Y_{t}\right)$ is a quotient of $\operatorname{ker}\left(\mathrm{d}^{4}\right) \subset H^{4}\left(W_{1}\right) \oplus H^{4}\left(W_{2}\right)$. Since $W_{1}$ and $W_{2}$ are merely log Calabi-Yau fourfolds, they have no holomorphic four-forms, so indeed 
$F^{4} \mathrm{Gr}_{4}=0$. For future use, also note that $F^{4} \cap \mathrm{W}_{3}=0$, as $\mathrm{Gr}_{3}$ has a Hodge structure of weight three which trivially has no $F^{4}$ part. It then also follows that $F^{4} \cap \mathrm{W}_{4}=0$.

Now even though $F^{4} \mathrm{Gr}_{4}=0$, it is not true that $\int_{f_{j}} \Omega_{0}$ vanishes. The reason is that $\mathrm{W}_{5} / \mathrm{W}_{3}$ is not simply a sum of graded pieces, but rather a non-trivial extension of mixed Hodge structures. So to recover $F^{4}\left(\mathrm{~W}_{5} / \mathrm{W}_{3}\right)$, we also need to study this extension class, denoted by $\operatorname{Ext}_{\mathrm{MHS}}^{1}\left(\mathrm{Gr}_{5}, \mathrm{Gr}_{4}\right)$.

Let us see this more explicitly for one of the simplest possible extensions, namely the extension of the constant Hodge structure $\mathbf{Z}(0)$ by $\mathbf{Z}(1)$. Explicitly, we have $H_{\mathbf{Z}}=\left\langle h^{0}, h^{1}\right\rangle$. The weight filtration is given by $\mathrm{W}_{-2} H=\left\langle h^{0}\right\rangle \subseteq W_{0}=H$, and the Hodge filtration is given by $F^{0} H_{\mathbf{C}}=\left\langle h^{1}-\log (q) h^{0}\right\rangle \subseteq H_{\mathbf{C}}$ for some $q \in \operatorname{Ext}_{\mathrm{MHS}}^{1}(\mathbf{Z}(0), \mathbf{Z}(1))=\mathbf{C}^{*}$. Note that $F^{0} \cap \mathrm{W}_{-2}=0$, much like $F^{4} \mathrm{Gr}_{4}=0$ above, since $F^{0}$ and $\mathrm{W}_{-2}$ are two distinct planes in $\mathbf{C}^{2}$, which intersect only at the origin. Nevertheless, if in analogy with above we denote $h^{1}-\log (q) h^{0}$ by $\Omega$, then we could write the period map as

$$
\Omega=h^{1} \int_{h_{1}} \Omega+h^{0} \int_{h_{0}} \Omega
$$

and we have $\int_{h_{0}} \Omega=-\log (q) \neq 0$.

The problem of characterizing extensions of mixed Hodge structures has been studied by Carlson [46]. Let us consider a general extension

$$
0 \rightarrow A \rightarrow C \rightarrow B \rightarrow 0
$$

If the mixed Hodge structure is separated, in the sense that the highest weight of $A$ is less than the lowest weight of $B$, then the generators of $\operatorname{Ext}_{\mathrm{MHS}}^{1}(B, A)$ are parametrized by the generalized complex torus

$$
\mathcal{J}^{0} \operatorname{Hom}(B, A) \equiv \operatorname{Hom}_{\mathbf{C}}(B, A) /\left(F^{0} \operatorname{Hom}(B, A)+\operatorname{Hom}_{\mathbf{Z}}(B, A)\right)
$$

Here $F^{0} \operatorname{Hom}(B, A)=\left\{\phi \in \operatorname{Hom}_{\mathbf{C}}(B, A) \mid \phi\left(F^{p} B\right) \subseteq F^{p} A\right\}$. Furthermore, in geometric situations we can usefully represent these maps as follows. Let $\sigma_{\mathbf{C}}$ : 
$B \rightarrow C$ be a section, i.e. a map such that $\sigma\left(F^{p} B\right) \subseteq F^{p} C$ but which does not preserve the integral structure. Let $r_{\mathbf{Z}}: C_{\mathbf{Z}} \rightarrow A_{\mathbf{Z}}$ be an integral retraction, which is defined as follows: take an integral basis $e^{i}$ for $A$, consider the dual basis $e_{i}$ for $A^{\vee}$, and lift it to $C^{\vee}$ using the surjection $C^{\vee} \rightarrow A^{\vee}$. Then we have $r_{\mathbf{Z}}(c)=\sum_{i}\left\langle c, e_{i}\right\rangle e^{i}$. This preserves the integral structure but not the Hodge structure. Using these maps, the extension classes may be represented as $\psi=r_{\mathbf{Z}} \circ \sigma_{\mathbf{C}}$. In the above example, this would give $\psi(\Omega)=h^{0} \int_{h_{0}} \Omega=-\log (q) h^{0}$.

We want to apply this result to our short exact sequence:

$$
0 \rightarrow \mathrm{Gr}_{4} \rightarrow \mathrm{W}_{5} / \mathrm{W}_{3} \rightarrow \mathrm{Gr}_{5} \rightarrow 0
$$

which is certainly separated. Let us first discuss an important special case, which arises in heterotic/ $F$-theory duality (see [47] and appendix $\mathrm{C}$ of [4]) and in IIB/ $F$ theory duality [7]. In these cases we have certain special divisors ("cylinders") $R_{1,2} \subset W_{1,2}$, intersecting the boundary in $R_{1,2} \cap Z=C_{1,2}$, which can be used to construct an isomorphism of Hodge structures appearing on the two sides. In the $E_{8} \times E_{8}$ case, we get an isomorphism

$$
H^{2}\left(C_{1}\right)_{\Lambda} \rightarrow H^{4}\left(W_{1}\right)_{\Lambda}
$$

and similarly for $W_{2}$. Here $H^{4}\left(W_{1}\right)_{\Lambda} \subset H^{4}\left(W_{1}\right)$ is the sublattice corresponding to the allowed G-fluxes in $F$-theory, and $H^{2}\left(C_{1}\right)_{\Lambda}$ is a sublattice which is defined as follows: we have an action of the $E_{8}$ Weyl group on the cohomology of $C_{1}$, and hence the cohomology of $C_{1}$ decomposes into isotypic pieces. The sublattice $H^{2}\left(C_{1}\right)_{\Lambda}$ corresponds to the eight-dimensional representation, obtained from the action of the Weyl group on the $E_{8}$ root lattice. To descend to $\mathrm{Gr}_{4}$, we further have to restrict this to $\operatorname{ker}\left(\mathrm{d}^{4}\right)$. Such classes end up in $H^{2}\left(C_{1}\right)_{\mathrm{van}}$, which is defined to be the kernel of the map $i_{1 *}: H^{2}\left(C_{1}\right) \rightarrow H^{2}(Z)$. One should be careful that the surface $C_{1}$ is not smooth. There are several ways for dealing with this, but in the present discussion we will ignore the singularities. There is a very similar story for the $S O(32)$ heterotic and IIB limits [7]. In that work we also take the singularities into account.

As before, we also have the isomorphism $\mathrm{Gr}_{5} \cong \operatorname{coker}\left(\mathrm{d}^{3}\right)$. In heterotic/Ftheory duality and IIb/F-theory duality, this is actually simply $H^{3}(Z)$. So ap- 
parently, our short exact sequence (4.25) is describing an extension of $H^{3}(Z)$ by a subgroup of $H_{\text {van }}^{2}\left(C_{1,2}\right)$.

Now there is indeed such an extension, and its $F^{4}$ part (or $F^{3}$ part after the isomorphisms, which shift the degree down) was described in some detail in section 9 of [33]. Namely if $H^{3}(C)=0$, which holds for example if $C$ is very ample, we have the following exact sequence for the relative cohomology group $H^{3}(Z, C)$ :

$$
0 \rightarrow H_{\text {van }}^{2}(C) \rightarrow H^{3}(Z, C) \rightarrow H^{3}(Z) \rightarrow 0
$$

Further restricting to $H^{2}(C)_{\Lambda}$, as we will implicitly assume in the following, the extension yields a subgroup of $H^{3}(Z, C)$. Then we can apply Carlson's prescription to get an explicit expression for the extension class. We take an integral basis $c^{j}$ for $H_{\text {van }}^{2}(C)$ mapping to $f^{j}$ in $\mathrm{Gr}_{4}$, and denote the dual basis of $H_{2, \text { van }}(C)$ by $c_{i}$. A lift to $H_{3}(Z, C)$ is given by choosing a set of three-chains $\Gamma_{j}$ such that $\partial \Gamma_{j}=c_{j}$. Note that such a lift is ambiguous up to classes in $H_{3}(Z, \mathbf{Z})$. We further take the holomorphic three-form $\Omega^{3,0}$ in $H^{3}(Z)$ and lift it to $H^{3}(Z, C)$, which can be done since it vanishes in $H^{2}(C)$. Then the homomorphism representing the $F^{3}$ part of the extension class is given by

$$
\psi=\sum_{j} c^{j} \psi_{j}=\sum_{j} c^{j} \int_{\Gamma_{j}} \Omega^{3,0}
$$

We recognize this as one of the ways to write the superpotential for 7-branes wrapped on $C \subseteq Z$, with worldvolume gauge flux given by $c^{j}$. As noted above $C$ is not smooth, but we will ignore the singularities here. It is shown in [33] that when $C$ is smooth, $\psi$ (or more precisely, any integral linear combination of the individual terms) has all the expected properties: it varies holomorphically in the complex structure moduli, and its critical locus (with respect to the complex structure moduli that keep $Z$ fixed) is the Noether-Lefschetz locus, where the worldvolume gauge flux is of Hodge type $(1,1)$. Up to a shift by a class inherited from $H^{2}(Z)$, which is always of type $(1,1)$ as $h^{2,0}(Z)=0$, a point on the critical locus thus determines the spectral data of a holomorphic $E_{8}$ bundle on $Z$. Also, by changing the bounding chains $\Gamma_{j}$, we see that $\psi_{j}$ is defined only modulo the periods of $\Omega^{3,0}$ over the lattice $H_{3}(Z, \mathbf{Z})$, as expected for a Chern-Simons-like 
functional. In the present context, this is a reflection of the Hodge structure being of mixed type.

In general we should probably not expect such nice divisors as $R_{1}$ and $R_{2}$ above. We will use a different description. Recall that despite the suggestive notation, $\Omega_{0}$ is not quite the holomorphic four-form on $Y_{t}$, rather it is the $F^{4}$ part with respect to the limiting mixed Hodge structure on $H^{4}\left(Y_{t}\right)$. However, there is a sense in which it corresponds to a $(4,0)$ form. For $t \neq 0$ the holomorphic $(4,0)$ form is a section of a line bundle $\mathcal{F}^{4}$ over the disk $\Delta_{t}$, which extends over the origin. Geometrically such a section determines a logarithmic $(4,0)$ form on the central fiber $Y_{0}$, up to periodic identifications of the residues.

Now we can try to relate this to the discussion of section 3 by using Steenbrink's description of the limiting mixed Hodge structure, which uses the logarithmic cohomology $H_{\log }^{4}\left(Y_{0}\right)$. The limiting mixed Hodge structure corresponds to the obvious Hodge filtration and a less obvious weight filtration on $H_{\log }^{4}\left(Y_{0}\right)$, as discussed in appendix A. The $F^{4}$ part is generated by the logarithmic $(4,0)$ form obtained above.

Let us look at the representing homomorphism for the extension (4.25), using this description of the limiting mixed Hodge structure. We take an integral basis $\tilde{f}^{j}$ for $\mathrm{Gr}_{4}$, take its dual $\tilde{f}_{j}$, and lift to $f_{j}$ in the dual of $\mathrm{W}_{5} / \mathrm{W}_{3}$. Geometrically, the $f_{j}$ correspond to cycles in $H_{4}\left(W_{1}\right)$ or $H_{4}\left(W_{2}\right)$, up to a linear combination of 'vanishing cycles.' Then the relevant part of the representing homomorphism is given by

$$
\psi=\sum_{j} \tilde{f}^{j} \int_{f_{j}} \Omega_{Y_{0}}^{4,0}
$$

The ambiguities by the vanishing cycles correspond to ambiguities by the periods $\int_{e_{i}} \Omega_{0}$ discussed previously.

Thus we have

$$
\psi_{j}=\int_{f_{j}} \Omega_{0}=\int_{f_{j}} \Omega_{Y_{0}}^{4,0}
$$

But the expressions above are precisely the integrals we previously encountered in section 3.2 , up to the usual ambiguity by $H_{3}(Z, \mathbf{Z})$. As we saw, we can rewrite them on $Z$ as $\int_{Z} \Omega^{3,0} \wedge \omega_{C S}$, see eg. equation (3.14). An alternative strategy is 
to note that $\mathrm{W}_{5} / \mathrm{W}_{3}$ is dual to $\mathrm{W}_{4}$ with respect to the polarization, so that we can equivalently study the extension class for $0 \rightarrow \mathrm{Gr}_{3} \rightarrow \mathrm{W}_{4} \rightarrow \mathrm{Gr}_{4} \rightarrow 0$.

In the final step, we want to lift $F^{4}\left(\mathrm{~W}_{5} / \mathrm{W}_{3}\right)$ to $F^{4} \cap \mathrm{W}_{5}$. Equivalently since $F^{4} \cap \mathrm{W}_{4}=0$ and since we have the exact sequence

$$
0 \rightarrow \mathrm{W}_{4} \rightarrow \mathrm{W}_{5} \rightarrow \mathrm{Gr}_{5} \rightarrow 0
$$

we can try to lift the extension class $\operatorname{Ext}_{\mathrm{MHS}}^{1}\left(\mathrm{Gr}_{5}, \mathrm{Gr}_{4}\right)$ to $\operatorname{Ext}_{\mathrm{MHS}}^{1}\left(\mathrm{Gr}_{5}, W_{4}\right)$. From the short exact sequence $0 \rightarrow \mathrm{Gr}_{3} \rightarrow \mathrm{W}_{4} \rightarrow \mathrm{Gr}_{4} \rightarrow 0$, we get the long exact sequence

$\ldots \rightarrow \operatorname{Ext}^{0}\left(\mathrm{Gr}_{5}, \mathrm{Gr}_{4}\right) \rightarrow \operatorname{Ext}^{1}\left(\mathrm{Gr}_{5}, \mathrm{Gr}_{3}\right) \rightarrow \operatorname{Ext}^{1}\left(\mathrm{Gr}_{5}, \mathrm{~W}_{4}\right) \rightarrow \operatorname{Ext}^{1}\left(\mathrm{Gr}_{5}, \mathrm{Gr}_{4}\right) \rightarrow 0$

Here we use the fact $\left([48]\right.$, proposition 3.35) that $\operatorname{Ext}_{\mathrm{MHS}}^{p}$ vanishes when $p \geq 2$. So we see that $\operatorname{Ext}_{M H S}^{1}\left(\mathrm{Gr}_{5}, \mathrm{Gr}_{4}\right)$ can always be lifted to $\operatorname{Ext}_{\mathrm{MHS}}^{1}\left(\mathrm{Gr}_{5}, \mathrm{~W}_{4}\right)$, but the choice of lift is parametrized by a class in $\operatorname{Ext}_{\mathrm{MHS}}^{1}\left(\mathrm{Gr}_{5}, \mathrm{Gr}_{3}\right)$. These extensions are indeed non-trivial. If $\mathrm{Gr}_{5}$ were one-dimensional, this is essentially our earlier example, the non-trivial extension of $\mathbf{Z}(0)$ by $\mathbf{Z}(1)$.

Let us try to write the representing homomorphism using the logarithmic $(4,0)$ form. We take a three-cycle $d_{k}$ in $H_{3}(Z, \mathbf{Z})$ and lift it to the dual of $\mathrm{W}_{5}$. We can represent this by a pair of four-chains $g_{k}=\left(c_{1, k}, c_{2, k}\right)$ in $W_{1}$ and $W_{2}$, such that $\partial c_{1, k}=-\partial c_{2, k}=d_{k}$. Then we have

$$
\int_{g_{k}} \Omega_{0}=\int_{g_{k}} \Omega_{Y_{0}}^{4,0}
$$

It is not completely clear how to write this as a simple expression, but at least we can argue that the expression can be localized on $Z$. To see this, suppose that we pick another lift $\tilde{c}_{1, k}$ with $\partial \tilde{c}_{1, k}=d_{k}$. Then $c_{1, k}-\tilde{c}_{1, k}$ is a closed fourcycle on $W_{1}$. Therefore modulo certain discrete ambiguities given by the periods $\int_{f_{j}} \Omega_{0}$ (and actually also $\int_{e_{i}} \Omega_{0}$ ), the expression is independent of the extension of the cycle away from $Z$. Furthermore we have already seen previously that these ambiguities can be rewritten as expressions on $Z$, so the result follows. We will write it informally as $\int_{g_{k}} \Omega_{0}=\int_{d_{k}} \Phi$. 
To summarize, let us collect all the pieces. The period map for the nilpotent orbit is of the form

$$
\Omega_{0}(t)=e^{i} \int_{d_{i}} \Omega^{3,0}+f^{j} \psi_{j}+g^{k}\left(\frac{1}{2 \pi i} \log (t) \int_{d_{k}} \Omega^{3,0}+\int_{d_{k}} \Phi\right)
$$

Geometrically, we think of $\int_{e_{i}} \Omega(t)$ as periods over the vanishing cycles, $\int_{f_{j}} \Omega(t)$ as periods over cycles in $H_{4}\left(W_{1}\right) \oplus H_{4}\left(W_{2}\right)$, and $\int_{g_{k}} \Omega(t)$ as periods over four-cycles which have to 'cross the neck.'

For comparison with expressions predicted by dualities, we define the variable

$$
T=\frac{1}{2 \pi i} \log (t)
$$

Then we have found (see (A.26) for a more precise statement) that for any semistable degeneration $Y_{t} \rightarrow Y_{0}=W_{1} \cup_{Z} W_{2}$ with $W_{1,2}$ log Calabi-Yau fourfolds, the flux superpotential $W=\frac{1}{2 \pi i} \int_{Y_{t}} \Omega^{4,0} \wedge \mathrm{G}$ is of the asymptotic form

$$
W=\int_{Z} \Omega^{3,0} \wedge H+\int_{Z} \Omega^{3,0} \wedge \omega_{C S}+T \int_{Z} \Omega^{3,0} \wedge \widetilde{H}+\int_{Z} \Phi \wedge \widetilde{H}+\mathcal{O}\left(T e^{2 \pi i T}\right)
$$

Here the first term comes from $F^{4} \mathrm{Gr}_{5}$; the second part comes from the $F^{4}$ part of the extension of $\mathrm{Gr}_{5}$ by $\mathrm{Gr}_{4}$, and the last part comes from $\log (t) N$ acting on $F^{4} \cap W_{5}$ and from the $F^{4}$ part of the extension of $\mathrm{W}_{5} / \mathrm{W}_{3}$ by $\mathrm{W}_{3}$. The exponential terms are corrections to the nilpotent orbit, which should be interpreted as instanton corrections. Note that the fluxes (or rather the combination $H+\omega_{C S}$ as discussed in section 3.2) in general do not take values in the full $H^{3}(Z, \mathbf{Z})$ lattice, but only in coker $\left(d^{3}\right)^{\perp}$, the sublattice which is orthogonal to the cokernel of $H^{3}\left(W_{1}\right) \oplus H^{3}\left(W_{2}\right) \rightarrow H^{3}(Z)$ with respect to the intersection pairing.

NOTE ADDED IN PROOF: The authors of 1404.7645 claim that the above formula (4.36) is inconsistent with formulas obtained in their work. However, we are considering $N^{2}=0$ stable degenerations to normal crossings unions of two smooth divisors, while they are considering the maximal unipotent monodromy limit and these limits are not comparable. Furthermore, the Gamma class appears in the large volume limit of the A-model, and again has no relevance for us. 
Up to one issue which we discuss below, we see that this reproduces known examples. In the heterotic $E_{8} \times E_{8}$ or $S O(32)$ limits of $K 3$ fibrations, the parameter $T$ is identified as a Kähler modulus associated to the elliptic fiber of the heterotic compactification, and the corrections are interpreted as coming from worldsheet instantons whose contribution is proportional to $\exp (2 \pi i T)$. Similarly in the Sen limit, $T$ is identified with axio-dilaton $\tau$ of type IIB $\left(\tau=i / g_{s}+a\right)$, and the corrections are interpreted as $D(-1)$-instantons, which do indeed give a contribution of the form $\exp (2 \pi i T)$.

The approach to the periods considered above is a generalization of the work of [10]. There are many other works on periods in heterotic/F-theory duality. For more computational approaches, see for example $[49,50,36,51,52,53,54$, $55,56,57]$.

Our analysis also gives further support for holography, i.e. the claim that the theory can be thought of as supported on the boundary $Z$. Moreover we see that the form of the Lagrangian is almost completely fixed from very little input. The only thing we don't know is exactly what CS theory we are dealing with. This clearly depends on the concrete model under consideration. From the example given earlier in the paper, it is clear that $S O(32)$ and $E_{8} \times E_{8}$ are not the only possible answers.

We have to make some remarks on the terms involving $\widetilde{H}$. The $E_{8} \times E_{8}$ heterotic string has only a single two-from field $B_{\mu \nu}$, so if we take the $E_{8} \times E_{8}$ limit then the superpotential is not expected to depend on a second tensor field. This is not a contradiction. The expression (4.36) really corresponds to all possible linear combinations of the periods. F-theory places restrictions on the allowed fluxes, namely they should be orthogonal to four-cycles contained in in the base $B_{3}$ or of the form $\pi^{-1}(C)$, where $C$ is a two-cycle in the base. This tends to place restrictions on G-fluxes coming from $H^{4}\left(W_{1}\right)$ and $H^{4}\left(W_{2}\right)$. If we had allowed such fluxes, then for example the Chern-Simons theory coming from the local $d P_{9}$-model would have a gauge group larger than $E_{8}$, as was checked carefully in [4]. However the G-fluxes which descend to $\widetilde{H}$ are of a slightly different form. They are Poincaré dual to four-cycles on $Y_{t}$ which asymptote to four-cycles on $Y_{0}$ of the form $c_{1}+c_{2}$, where $c_{1,2}$ are four-chains in $W_{1,2}$ such that $\partial c_{1}=-\partial c_{2}=$ $b \in H_{3}(Z, \mathbf{Z})$. It is not clear to us why such fluxes would be disallowed, but we 
have not checked it precisely.

\subsection{The Kähler potential}

One may similarly compute the Kähler potential on the nilpotent orbit. In the following, we will compare this with the $E_{8} \times E_{8}$ limit. The Kähler potential is not protected, so it would be remarkable if we would get an exact match. This is not the case, but nevertheless we can match the main terms.

The Kähler potential in F-theory is of the following form:

$$
\mathcal{K}_{F}=-\log \left[\int_{Y} \Omega^{4,0} \wedge \overline{\Omega^{0,4}}\right]-2 \log \mathcal{V}_{B_{3}}+\mathcal{K}_{C_{3}}
$$

We set the four-dimensional Planck scale $M_{P l}$ equal to one. Note that up to a constant, $\int_{Y_{t}} \Omega(t) \wedge \overline{\Omega(t)}$ is simply $Q(\Omega, \bar{\Omega})$, where $Q$ is the polarization on $H^{4}\left(Y_{t}\right)$. By general properties of the monodromy, we have $Q(N a, N b)=-Q\left(a, N^{2} b\right)=$ 0 for any forms $a, b$. For simplicity, we will assume that the polarization is simply the sum of the polarizations on the graded pieces. In particular, $Q(\cdot, N \cdot)$ corresponds to the polarization of the weight three Hodge structure on $\mathrm{Gr}_{3} \simeq$ $H^{3}(Z, \mathbf{Z})$. Then we have

$$
Q(\Omega(t), \bar{\Omega}(t)) \sim \mathrm{T} Q_{3}+Q_{4}
$$

where $\mathrm{T}=\operatorname{Im}\left(\frac{1}{2 \pi i} \log (t)\right), Q_{3}=i \int_{Z} \Omega^{3,0} \wedge \overline{\Omega^{3,0}}$ and $Q_{4}$ is the polarization on $\mathrm{Gr}_{4}$, evaluated on the image of $\Omega_{0}$ under the projection $H^{4}\left(Y_{t}\right) \rightarrow \mathrm{Gr}_{4}$. The Kähler potential for the nilpotent orbit is then given by

$$
\mathcal{K}_{\Omega(t)} \sim-\log \left[\mathrm{T} Q_{3}+Q_{4}\right] \sim-\log (\mathrm{T})-\log \left[Q_{3}\right]+\frac{Q_{4}}{\mathrm{~T} Q_{3}}+\mathcal{O}\left(\mathrm{T}^{-2}\right)
$$

Furthermore using the isomorphism $\mathrm{Gr}_{4} \rightarrow H_{\mathrm{v}}^{2}\left(C_{1}\right)_{\Lambda} \oplus H_{\mathrm{v}}^{2}\left(C_{2}\right)_{\Lambda}$, we see that up to a constant, $Q_{4}$ is mapped to the natural polarization on the second cohomology of the spectral covers $C_{1}$ and $C_{2}$.

Now let us take a closer look at the Kähler moduli. In heterotic/F-theory duality, the $F$-theory base $B_{3}$ is a $\mathbf{P}^{1}$-fibration over a surface $B_{2}$. The volume is 
then given by

$$
\mathcal{V}_{B_{3}}=\frac{1}{2} \mathrm{FB}^{i} \mathrm{~B}^{j} d_{i j}+\frac{1}{2} \mathrm{~F}^{2} \mathrm{~B}^{i} d_{i j} n^{j}+\frac{1}{6} \mathrm{~F}^{3} d_{i j} n^{i} n^{j}
$$

where $\mathbf{F}$ is the volume of the $\mathbf{P}^{1}$-fiber, $\mathrm{B}^{i} d_{i j}$ are volumes of two-cycles in $B_{2}, n^{i}$ is the class of the normal bundle of $B_{2}$ in $B_{3}$, and $d_{i j}$ is the intersection form on $B_{2}$. We first need to rewrite this in terms of the Kähler moduli, which are given by $\mathrm{S}=\partial \mathcal{V}_{B_{3}} / \partial \mathrm{F}$ and $\mathrm{T}^{i}=d^{i j} \partial \mathcal{V}_{B_{3}} / \partial \mathrm{B}^{j}$. If we further assume that $B_{3}=B_{2} \times \mathbf{P}^{1}$ is a direct product, then the terms involving $n^{i}$ in (4.40) are absent and we can easily rewrite this in terms of the Kähler moduli as $\mathcal{V}_{B_{3}}=\mathrm{S}^{1 / 2}\left(\mathrm{~T}^{i} \mathrm{~T}^{j} d_{i j}\right)^{1 / 2}$. Then we have

$$
K_{F} \sim-\log (\mathrm{S})-\log (\mathbf{T})-\log \left(\mathrm{T}^{i} \mathrm{~T}^{j} d_{i j}\right)-\log \left[Q_{3}\right]+\frac{Q_{4}}{\mathrm{~T} Q_{3}}+\mathcal{K}_{\mathrm{C}_{3}}+\mathcal{O}\left(\mathrm{T}^{-2}\right)
$$

where we omitted the additional terms in (4.39) and (4.40). We also have $\partial \bar{\partial} \mathcal{K}_{\mathrm{C}_{3}} \sim \int_{Y} \delta \mathrm{C}_{3} \wedge * \delta \mathrm{C}_{3}$, but for Calabi-Yau four-folds there are usually no moduli of the form $\delta \mathrm{C}_{3}$ as generically $H^{3}(Y)=0$.

On the $E_{8} \times E_{8}$ heterotic side, the Kähler potential is of the form

$$
\mathcal{K}_{\text {het }} \sim-\log (\mathrm{S})-\log \left(\mathcal{V}_{Z}\right)-\log \left[i \int_{Z} \Omega^{3,0} \wedge \overline{\Omega^{3,0}}\right]+\mathcal{K}_{A}
$$

where $\mathcal{K}_{A}$ denote the Kähler potential for the bundle moduli. The volume $\mathcal{V}_{Z}$ can be written as

$$
\mathcal{V}_{Z}=\frac{1}{2} \mathrm{~T}^{i} \mathrm{~T}^{j} d_{i j}+\frac{1}{2} \mathrm{~T}^{2} \mathrm{~T}^{i} d_{i j} k^{j}+\frac{1}{6} \mathrm{~T}^{3} d_{i j} k^{i} k^{j}
$$

The potential for bundle moduli is harder to write, but we can easily write the Kähler metric:

$$
\partial \bar{\partial} \mathcal{K}_{A} \sim \frac{1}{\mathcal{V}_{Z}} \int_{Z} \operatorname{Tr}(\delta A \wedge \overline{\delta A}) \wedge J \wedge J \sim \frac{1}{\overline{\mathrm{T}}} \int_{B_{2}} \operatorname{Tr}_{E}\left(\delta A_{\nu} \wedge \overline{\delta A}_{\nu}\right)+\mathcal{O}\left(\mathrm{T}^{-2}\right)
$$

Here $\delta A_{\nu}$ denotes the component of $\delta A$ along the elliptic curve $E$, which correspond to normal bundle valued moduli of the spectral cover. We can map them 
to $(2,0)$ forms on the spectral cover by contracting with $\Omega_{Z}^{3,0}$. Thus we can write

$$
\frac{1}{\overline{\mathrm{T}}} \int_{B_{2}} \operatorname{Tr}_{E}\left(\delta A_{\nu} \wedge \overline{\delta A}_{\nu}\right) \sim \frac{1}{\mathrm{~T} Q_{3}} \int_{B_{2}} \operatorname{Tr}\left(\delta \Phi^{2,0} \wedge \overline{\delta \Phi^{2,0}}\right)
$$

Abelianizing $\delta \Phi^{2,0}$ we get $(2,0)$ forms on the spectral cover, which get mapped to variations $\delta \Omega_{0}$ sitting in $\mathrm{Gr}_{4}$ under the cylinder map. So we see that this describes the metric for complex structure moduli of the spectral cover, and should be compared with $\partial \bar{\partial} Q_{4} / \mathrm{T} Q_{3}$ on the $F$-theory side.

Comparing $\mathcal{K}_{F}$ with $\mathcal{K}_{\text {het }}$, we see that the main terms match qualitatively, without doing any Kähler transformations. It might be interesting to do the comparison more carefully, as this gives some insight into the subleading terms which might be useful for the purpose of moduli stabilization.

In the IIb limit one can do a similar comparison. It is in fact more straightforward as the potentials for the Kähler moduli can be compared more easily.

\subsection{Euler character}

A number of additional properties of $Y_{t}$ can be deduced from the degeneration limit. For example, the Euler character or more generally Chern classes of $Y_{t}$ can be computed using Chern-Schwartz-Macpherson classes in the degeneration limit. Here we briefly consider the Euler character, which plays a role in tadpole cancellation. Recall that the cohomology groups of the smooth fibers agree with the logarithmic de Rham cohomology groups of the central fiber. Therefore if $Y_{t} \rightarrow Y_{0}=W_{1} \cup_{Z} W_{2}$, we have

$$
\chi\left(Y_{t}\right)=\sum_{k}(-1)^{k} H_{\log }^{k}\left(Y_{0}\right)=\chi\left(W_{1}\right)+\chi\left(W_{2}\right)-2 \chi(Z)
$$

Note that this differs from the topological Euler character of $Y_{0}$. The extra factor of $-\chi(Z)$ comes from the logarithmic forms that have a pole along $Z$, see equation (A.24).

We consider the following application. Suppose that we use the Tate form of the Weierstrass fibration to engineer an elliptic fibration with interesting singularities along a divisor $S \subseteq B_{3}$. The Euler character of the resolved $Y$ will depend 
on the singularities, and one may ask how it changes when we put different singularities along $S$. When $Y$ has a heterotic dual, we can deduce the answer from heterotic/F-theory duality. It was observed in section 2.3 of [58] that the same formula appears to hold more generally, when there is no heterotic dual (i.e. $Y$ does not admit a K3 fibration).

From the perspective of the present paper, this observation is a corollary of the result (4.46) together with the degeneration limit of section 2. When the singularities along $S$ fit in $E_{8}$, we can use our degeneration limit to move the singularities to $W_{1}$. But we already know $\chi\left(W_{1}\right)-\chi(Z)$ from heterotic/F-theory duality. Namely, it should agree with $\int_{S} \operatorname{ch}_{2}(V)-\frac{1}{2} \operatorname{ch}_{2}(T Z)$, which was computed in [59], upon setting $\gamma=0$ in their formulae (as this part gets mapped to the G-flux).

\subsection{Bulk versus boundary}

We would like to end with a few more comments.

Perhaps one of the most intriguing aspects of this paper is the interplay between the theory in the bulk and the theory on the boundary. For the $E_{8} \times E_{8}$ and $S O(32)$ degeneration limits of the $K 3$-surface, it has long been known that the heterotic theory may be thought of as living on the boundary (the normal crossing divisor), but given the lack of examples it was less than clear how general this is. In sections 3 and 4 we have given arguments that this should be true in more general degeneration limits, and in [7] we give another concrete example of this phenomenon. It would be interesting to understand the general boundary theories in more detail.

Kawamata/Namikawa also allow the normal crossing divisor to have multiple components $Z_{i}$ which intersect. This leads us to consider defects in the boundary theory. The defect theories should be very interesting. As indicated in section 3, we expect to find a kind of holomorphic generalization of WZW models on such defects. One should be able to deduce some of its properties from an analysis of the limiting mixed Hodge structure, similar to sections 4.2 and 4.3 .

Log geometry plays a central role in the mirror symmetry program of Gross and Siebert [60], and their philosophy of gluing Calabi-Yau manifolds from more 
elementary pieces is similar to the one in this paper, although their singular Calabi-Yau spaces are not necessarily of normal crossing type. It would be very interesting to understand the holographic aspects of their work.

Finally, the superpotential still receives corrections that are non-perturbative in the Kähler moduli, due to Euclidean brane configurations. In type II these come from $D$-instantons, in $F$-theory they come from $M 5$-instantons and in the heterotic string they come from worldsheet instantons and NS5-instantons. They are all related through heterotic/ $F$-theory/type II duality. It is hard to escape the impression that there must be some underlying variation of Hodge structure problem that encodes all instanton corrections to the superpotential in $4 d$ compactifications with $N=1$ supersymmetry. It would be extremely interesting to elucidate this.

Acknowledgements: We are grateful to P. Aluffi, A. Clingher, J. Kollár, Y. Kawamata, T. Pantev, R. Thomas, B. Siebert, and E. Witten for discussions and correspondence related to this project. RD acknowledges partial support by NSF grants 0908487, 0636606 and DMS 1304962. SK acknowledges partial support by NSF grants DMS-05-55678 and DMS-12-01089 in addition to the hospitality of the University of Pennsylvania. The research of MW is supported by a Heisenberg grant from the DFG.

\section{Appendix A: Mixed Hodge structures and monodromy}

In this appendix we briefly review some aspects of Hodge theory that will help us understand a semi-stable degeneration limit. There are many books and reviews available on this material, see for example [61, 62] and [48].

Recall that on a compact Kähler manifold $X$ of complex dimension $n$, the cohomology groups admit a Hodge decomposition

$$
H^{k}(X, \mathbf{C})=\sum_{p+q=k} H^{p, q}(X) .
$$

Let us denote the Kähler form of $X$ by $J$, which we may take to be a class 
in $H^{2}(X, \mathbf{Z})$. The primitive part of the cohomology, denoted by $P H^{k}(X, \mathbf{Z})$, is defined to be the kernel of $\wedge J^{n-k+1}: H^{k}(X, \mathbf{Z}) \rightarrow H^{2 n-k+2}(X, \mathbf{Z})$. Then we have a further decomposition of the cohomology, the Lefschetz decomposition:

$$
H^{k}(X, \mathbf{Q})=\bigoplus_{m} J^{m} \cdot P H^{k-2 m}(X, \mathbf{Q})
$$

Since $J$ is of type $(1,1)$, the interesting part of the information is contained in the primitive part of the cohomology, and we will often implicitly assume that we are restricting to the primitive part.

We further have a polarization, i.e. a non-degenerate bilinear form

$$
Q(a, b)=(-1)^{k(k-1) / 2} \int_{X} a \wedge b \wedge J^{n-k}
$$

which is symmetric for $k$ even and anti-symmetric for $k$ odd. It satisfies the following two relations, which are called the Hodge-Riemann bilinear relations: we have $Q\left(H^{p, q}, H^{p^{\prime}, q^{\prime}}\right)=0$ unless $p+p^{\prime}=q+q^{\prime}=k$, and $i^{p-q} Q(a, \bar{a}) \geq 0$ if $a \in P H^{p, q}$.

We will be interested in period integrals on $X$. The periods themselves depend on some redundant information, as we have to make a non-canonical choice of basis. A more invariant way to state it is that we are interested in how the Hodge decomposition $\bigoplus H^{p, q}(X)$ of $H^{k}(X, \mathbf{C})$ varies in families. However the $H^{p, q}(X)$ individually do not vary holomorphically even if $X$ lives in a holomorphic family.

Let $\pi_{\mathcal{X}}: \mathcal{X} \rightarrow B$ denote a smooth holomorphic family, with fibers $X_{t}$ for $t \in B$. On each fiber $X_{t}$ we have the cohomology lattice $H^{k}\left(X_{t}, \mathbf{Z}\right)$. As we vary $t \in B$, these lattices fit together in a local system $\mathcal{L}^{k}$ over $B$. Mathematically, we have $\mathcal{L}^{k}=R^{k} \pi_{\mathcal{X} *} \mathbf{Z}$. Similarly, for each fiber $X_{t}$ we have a vector space $H^{k}\left(X_{t}, \mathbf{C}\right)$. As we vary $t \in B$, these vector spaces fit together in a vector bundle $\mathcal{H}^{k} \rightarrow B$, which is just the complexification $\mathcal{H}^{k}=\mathcal{L}^{k} \otimes \mathcal{O}_{B}$. The vector bundle $\mathcal{H}^{k} \rightarrow B$ is flat and holomorphic. It has a natural flat Gauss-Manin connection $\nabla$, obtained by requiring that the local sections induced from local sections of $\mathcal{L}^{k}$ are flat.

Now we may put a decreasing filtration $\mathcal{F}^{\bullet}$ on the bundle $\mathcal{H}^{k}$, by putting the 
following decreasing filtration on each of the fibers:

$$
0 \subseteq F^{k} \subseteq F^{k-1} \subseteq \ldots \subseteq F^{0}=H^{k}\left(X_{t}, \mathbf{C}\right), \quad F^{p} \equiv \bigoplus_{q \geq p} H^{q, k-q}\left(X_{t}\right)
$$

Note that we may recover $H^{p, q}$ as $H^{p, q}\left(X_{t}\right)=F^{p} \cap \bar{F}^{q}$, so we did not lose any information by focusing on the filtration. Although the $H^{p, q}$ generally do not vary holomorphically, the subbundles $\mathcal{F}^{p} \rightarrow B$ with fibers $F^{p} \subset H^{k}\left(X_{t}, \mathbf{C}\right)$ do vary holomorphically.

The filtration satisfies the following properties. Let us denote $H_{\mathbf{Z}}=H^{k}\left(X_{t}, \mathbf{Z}\right)$. Then $F^{\bullet}$ is a $k$-step decreasing filtration of $H_{\mathbf{C}}=H_{\mathbf{Z}} \otimes \mathbf{C}$, such that $H_{\mathbf{C}} \cong$ $F^{p} \oplus \bar{F}^{k-p+1}$ for all $p$. We also have a bilinear form $Q(\cdot, \cdot)$ with the properies listed previously. As we vary $t \in B$, this yields a local system $\mathcal{L}^{k}$ over $B$ and a decreasing filtration $\mathcal{F}^{\bullet}$ of $\mathcal{H}^{k}=\mathcal{L}^{k} \otimes \mathcal{O}_{B}$, such that each $\mathcal{F}^{p}$ is a holomorphic vector bundle. Furthermore, the $\mathcal{F}^{p}$ behave nicely with respect to the Gauss-Manin connection, namely they satisfy Griffiths transversality

$$
\nabla: \mathcal{F}^{p} \rightarrow \mathcal{F}^{p+1} \otimes \Omega_{B}^{1}
$$

The data $\left(B, \mathcal{L}^{k}, \mathcal{F}^{\bullet}, Q(\cdot, \cdot), \nabla\right)$ satisfying the properties discussed above defines a polarized variation of Hodge structure of weight $k$.

A filtration in a fixed vector space is classified by a point in the flag manifold $\mathbf{F}\left(n_{k}, . ., n_{0}\right)$, where $n_{p}=\operatorname{dim} F^{p}$. After pullback to the universal cover $\rho: \tilde{B} \rightarrow B$, the bundle $\rho^{*} \mathcal{H}^{k}$ becomes trivial. Fixing a reference point in $\tilde{B}$ over a point $t \in B$, the Gauss-Manin connection gives an explicit isomorphism $\sigma: \rho^{*} \mathcal{H}^{k} \simeq$ $H^{k}\left(X_{t}, \mathbf{Z}\right) \otimes \mathcal{O}_{\tilde{B}}$. Using this isomorphism we get a holomorphic period mapping

$$
\Pi: \tilde{B} \rightarrow \mathbf{F}\left(n_{k}, \ldots, n_{0}\right),
$$

by associating to each $s \in \tilde{B}$ the flag $\mathcal{F}_{\rho(s)}^{\bullet}$, identified with a flag in $H^{k}\left(X_{t}, \mathbf{Z}\right)$ via $\sigma$. If the Hodge structure is polarized, its image is contained in the period domain, which consists of the flags which satisfy the Hodge-Riemann bilinear relations, i.e. $Q\left(F^{p}, F^{k-p+1}\right)=0$, and $i^{p-q} Q(a, \bar{a}) \geq 0$ if $a \in P H^{p, q}$. 
By introducing a basis of integral cycles of $X$, we can write the mapping $\Pi$ in coordinate form in terms of period integrals, but this explicit form obviously depends on a non-canonical choices. Continuing to fix our reference point $t \in B$, we pick a basis $e_{i} \in H_{k}\left(X_{t}, \mathbf{Z}\right)$ and its dual basis $e^{i} \in H^{k}\left(X_{t}, \mathbf{Z}\right)$. We use the Gauss-Manin connection to extend this basis away from $t$ by parallel transport. Globally, these bases becomes multi-valued over $B$ but single-valued over $\tilde{B}$. We also need to introduce a basis for the $\mathcal{F}^{p}$. Since the $\mathcal{F}^{p}$ are holomorphic vector bundles, locally on an open subset $U \subset B$ we can trivialize these bundles by choosing a holomorphic frame. It is convenient to adapt this basis to the filtration, i.e. we require $\left\{\omega_{1}, . ., \omega_{n_{k}}\right\}$ to project to a holomorphic frame for $\mathcal{F}^{k},\left\{\omega_{n_{k}+1}, . ., \omega_{n_{k-1}}\right\}$ to project to a frame for $\mathcal{F}^{k-1} / \mathcal{F}^{k}$, etc. Then the period mapping associates to each $t \in U$ the explicit flag

$$
0 \subseteq\left\langle x_{i, 1} e^{i}, \ldots, x_{i, n_{k}} e^{i}\right\rangle \subseteq\left\langle x_{i, 1} e^{i}, \ldots, x_{i, n_{k-1}} e^{i}\right\rangle \subseteq \ldots \subseteq H^{k}\left(X_{t}, \mathbf{C}\right)
$$

Here

$$
x_{i, r}=\int_{e_{i}} \omega_{r}
$$

and $\langle\ldots\rangle$ denotes the linear subspace of $H^{k}\left(X_{t}, \mathbf{C}\right)$ generated by the vectors between the brackets.

We are interested in degenerating a Hodge structure with some weight $k$. It turns out that in the limit the Hodge structure is not necessarily of pure type, but may contain pieces with higher or lower weight than $k$. This leads to some extra structure in the form of an increasing weight filtration $W_{\bullet}$ of $H^{k}(X, \mathbf{Q})$ :

$$
0 \subseteq W_{0} \subseteq \ldots \subseteq W_{i} \subseteq \ldots \subseteq W_{2 k}=H^{k}(X, \mathbf{Q})
$$

The filtrations $W_{\bullet}$ and $F^{\bullet}$ define a mixed Hodge structure when the weight filtration is compatible with the Hodge filtration $\mathcal{F}^{\bullet}$, in the sense that the induced filtrations $\mathcal{F}_{i}^{\bullet}$ on the graded pieces

$$
\mathrm{Gr}_{i}^{W}=W_{i} / W_{i-1}
$$

satisfy the axioms of a Hodge structure of weight $i$. By duality between homology and cohomology, we get an induced filtration on the homology, whose weights are 
the negatives of the weights on cohomology. Here we must use the conventional duality $H_{*}=\operatorname{Hom}\left(H^{*}, \mathbf{Z}\right)$, not Poincaré duality, which tends to fail on singular spaces.

It has been shown by Deligne that the cohomology of any variety $X$ canonically carries such a mixed Hodge structure. There are two basic cases: if $X$ is complete but possibly singular, then $W_{i}=H^{k}(X, \mathbf{Q})$ for $i \geq k$. If on the other hand $X$ is smooth but not necessarily complete, then $W_{i}=0$ for $i<k$.

Let us now suppose that we have a one-parameter semi-stable degeneration, i.e. we have a one-parameter family $\pi_{\mathcal{X}}: \mathcal{X} \rightarrow \Delta$ with fibers $X_{t}$, such that the total space is smooth and the central fiber is reduced and has at most normal crossing singularities. There are two mixed Hodge structures that we can associate to this set-up. The first is the mixed Hodge structure of the central fiber $X_{0}$, and the second comes from the monodromy weight filtration. Let us discuss each of these in turn.

First we consider the mixed Hodge structure of the central fiber. Recall that $X_{0}$ is a complete and reduced variety with only normal crossing singularities. The weight filtration in this case can be read of from the Mayer-Vietoris spectral sequence. Let us suppose that $X_{0}$ decomposes into irreducible pieces as $X_{0}=$ $D_{1} \cup \ldots \cup D_{N}$. We set $D_{i_{1} \ldots i_{q}}=D_{i_{1}} \cap \ldots \cap D_{i_{q}}$ and define the codimension $q$ stratum as

$$
X_{0}^{[p]}=\coprod_{1 \leq i_{1}<\ldots<i_{p} \leq N} D_{i_{1} \ldots i_{p}}
$$

where $\amalg$ denotes disjoint union. Now let us define

$$
E_{0}^{p, q} \equiv \mathcal{A}^{q}\left(X_{0}^{[p+1]}\right)
$$

where $\mathcal{A}^{\bullet}$ denotes De Rham cohomology. As usual, we have the exterior derivative $d: E_{0}^{p, q} \rightarrow E_{0}^{p, q+1}$. In addition, we have a second differential $\delta: E_{0}^{p, q} \rightarrow E_{0}^{p+1, q}$, which is the restriction map given by

$$
\delta \phi\left(D_{i_{1} \ldots i_{p+1}}\right)=\sum_{a}(-1)^{a} \phi\left(D_{i_{0} \ldots \hat{i}_{a} \ldots i_{p+1}}\right)
$$


Then we have $d^{2}=\delta^{2}=d \delta+\delta d=0$, so the data $\left(E_{0}^{\boldsymbol{\bullet}, \bullet}, d, \delta\right)$ yields a double complex. It can be shown that this spectral sequence converges to $H^{k}\left(X_{0}, \mathbf{R}\right)$.

Now we can use this to define a filtration:

$$
W_{i}=\bigoplus_{r \leq i} E_{0}^{\bullet, r}
$$

This descends to a weight filtration on $H^{k}\left(X_{0}, \mathbf{R}\right)$. Similarly we define a Hodge filtration as $F^{p}=\bigoplus_{r, s} F^{p} E_{0}^{r, s}$, which descends to $H^{k}\left(X_{0}, \mathbf{C}\right)$.

Let us discuss this in more detail for the main case of interest in this paper, where we have $X_{0}=W_{1} \cup_{Z} W_{2}$. Then we have

$$
E_{1}^{0, k}=H^{k}\left(W_{1}\right) \oplus H^{k}\left(W_{2}\right), \quad E_{1}^{1, k}=H^{k}(Z),
$$

with all other terms vanishing. The non-zero differentials are the restriction maps

$$
d_{1}^{0, k}: H^{k}\left(W_{1}\right) \oplus H^{k}\left(W_{2}\right) \rightarrow H^{k}(Z)
$$

and the spectral sequence degenerates at $E_{2}$. This gives a short exact sequence

$$
0 \rightarrow \operatorname{coker}\left(d_{1}^{0, k-1}\right) \rightarrow H^{k}\left(X_{0}\right) \rightarrow \operatorname{ker}\left(d_{1}^{0, k}\right) \rightarrow 0
$$

which corresponds to the weight filtration

$$
0 \subseteq W_{k-1} \subseteq W_{k}=H^{k}\left(X_{0}, \mathbf{R}\right)
$$

with $\operatorname{Gr}_{k} H^{k}\left(X_{0}\right) \cong \operatorname{ker}\left(d_{1}^{0, k}\right)$ and $\operatorname{Gr}_{k-1} H^{k}\left(X_{0}\right) \cong \operatorname{coker}\left(d_{1}^{0, k-1}\right)$. In fact, in this example it is simpler to read off the weight filtration directly from the long exact sequence version of Mayer-Vietoris:

$$
\ldots \rightarrow H^{k-1}(Z) \rightarrow H^{k}\left(W_{1} \cup_{Z} W_{2}\right) \rightarrow H^{k}\left(W_{1}\right) \oplus H^{k}\left(W_{2}\right) \rightarrow H^{k}(Z) \rightarrow \ldots
$$

We see that the graded pieces $\mathrm{Gr}_{k-1}$ and $\mathrm{Gr}_{k}$ should be thought of as a quotient or a subspace of the cohomology groups of $Z$ and $W_{1} \amalg W_{2}$ respectively. These 
are smooth and complete spaces, which is why they each carry a pure Hodge structure. This is in some sense the general picture: we find a suitable stratification to construct a weight filtration, so that the graded pieces can be though of as the Hodge structure of a complete non-singular variety, and hence carry a pure Hodge structure.

The second mixed Hodge structure associated to our semi-stable degeneration comes from the monodromy. As we circle around the origin $t=0$, the cycles in $H_{k}\left(X_{t}, \mathbf{Z}\right)$ get rearranged. This is expressed as an automorphism $M$ of $H^{k}\left(X_{t}, \mathbf{Z}\right)$ called the monodromy transformation. It is known for our semi-stable degeneration that the monodromy is unipotent. We also introduce the logarithm $N=\log (M)$, which is nilpotent since $M$ is unipotent.

The operator $N$ should be thought of as an analogue of the Lefschetz operator (cup product with the Kähler class). As such it gives rise to a kind of Lefschetz decomposition on $H^{k}\left(X_{t}, \mathbf{Q}\right)$, which in turn gives the weight filtration. The monodromy weight filtration is completely determined by the following two properties. The log of the monodromy matrix has degree -2 (i.e. $N \cdot W_{i} \subset W_{i-2}$ ), and $N^{j}$ gives an isomorphism $N^{j}: G r_{k+j} \rightarrow G r_{k-j}$. For example, if $N^{2}=0$ but $N \neq 0$, then the monodromy weight filtration simply takes the form

$$
0 \subseteq \operatorname{im}(N) \subseteq \operatorname{ker}(N) \subseteq H^{k}\left(X_{t}, \mathbf{Q}\right)
$$

i.e. we have

$$
W_{k-1}=\operatorname{im}(N), \quad W_{k}=\operatorname{ker}(N), \quad W_{k+1}=H^{k}\left(X_{t}, \mathbf{Q}\right)
$$

In the polarized case, we further have $Q(N a, b)+Q(a, N b)=0$. The polarization $Q$ of $H^{k}\left(X_{t}, \mathbf{Z}\right)$ yields a natural polarization on the graded pieces, i.e. $Q\left(\cdot, N^{j} \cdot\right)$ on $\operatorname{Gr}_{k+j}$. For example in the case $N^{2}=0, N \neq 0$ above, we have $(x, y) \rightarrow Q(x, N y)$ for $x, y \in H^{k}\left(X_{t}, \mathbf{Q}\right) / \operatorname{ker}(N)$, and we have $(x, y) \rightarrow Q(\tilde{x}, y)$ for $x, y \in \operatorname{im}(N)$, where $\tilde{x}$ is a lift from $\operatorname{im}(N)$ to $H^{k}\left(X_{t}, \mathbf{Q}\right)$ with $N \tilde{x}=x$.

So we have now used the monodromy to put a weight filtration on $H^{k}\left(X_{t}, \mathbf{Q}\right)$. But this weight filtration is not compatible with the Hodge filtration on $X_{t}$, because $X_{t}$ is smooth and so the Hodge structure is of pure type. However it has 
been shown by Schmid that the limiting Hodge filtration

$$
\mathcal{F}_{\infty}^{\bullet}=\lim _{t \rightarrow 0} e^{-\frac{1}{2 \pi i} \log (t) N} \mathcal{F}^{\bullet}
$$

exists, and together with the monodromy weight filtration it does define a mixed Hodge structure. It is called the limiting mixed Hodge structure.

There is an alternative description of the limiting mixed Hodge structure due to Steenbrink [12], which uses the logarithmic cohomology groups of $X_{0}$. We discuss this a bit more explicitly for $X_{0}=W_{1} \cup_{Z} W_{2}$. As in section 2.1 we use the short-hand notation $H_{\log }^{k}\left(X_{0}\right)=\mathbb{H}^{k}\left(X_{0}, \Omega_{X_{0}}^{\bullet}(\log Z)\right)$. The spectral sequence for $\mathbb{H}^{k}$ degenerates at the $E_{1}$ term, so the Hodge decomposition is the obvious one, $H_{\log }^{k}\left(X_{0}\right)=\sum_{k=p+q} H^{p}\left(X_{0}, \Omega^{q}(\log Z)\right)$. The weight filtration can be obtained as follows. We have the short exact sequence

$$
0 \rightarrow \Omega_{X_{0}}^{p} \rightarrow \Omega_{X_{0}}^{p}(\log Z) \stackrel{\text { res }}{\rightarrow} \Omega_{Z}^{p-1} \rightarrow 0
$$

where 'res' denotes the Poincaré residue map. This gives the long exact sequence

$$
\ldots \rightarrow H^{k-2}(Z) \rightarrow H^{k}\left(X_{0}\right) \rightarrow H_{\log }^{k}\left(X_{0}\right) \rightarrow H^{k-1}(Z) \rightarrow \ldots
$$

where all the maps are compatible with the Hodge structure, and the coboundary map $H^{k-2}(Z) \rightarrow H^{k}\left(X_{0}\right)$ is a Gysin map. We find that $\mathrm{W}_{k+1}=H_{\log }^{k}\left(X_{0}\right)$ and $\mathrm{W}_{k}=H^{k}\left(X_{0}\right) / \operatorname{Im}\left(H^{k-2}(Z)\right)$. From the Mayer-Vietoris sequence for $X_{0}$ (A.19) we further get $\mathrm{W}_{k-1}=\operatorname{coker}\left(\mathrm{d}^{k-1}\right)$ as before.

Given a limiting mixed Hodge structure, we can define the nilpotent orbit as

$$
\mathcal{O}=\left\{e^{\frac{1}{2 \pi i} \log (t) N} \mathcal{F}_{\infty}^{\bullet} \mid t \in \Delta\right\}
$$

The nilpotent orbit may be thought of as a kind of perturbative approximation to the periods in the limit $t \rightarrow 0$. The precise statement is the content of the nilpotent orbit theorem of Schmid [14]. As before let $\Pi$ denote the period mapping from the upper half plane (the universal cover of the punctured disc) to the period domain of the Hodge structure. Define $T=\frac{1}{2 \pi i} \log (t)$, the usual coordinate on 
the upper half plane. Then for any reasonable metric $d$ on the period domain, there are constants $A, B$ such that for $\operatorname{Im}(T) \geq A>0$ we have

$$
d(\Pi(\mathcal{O}), \Pi(\mathcal{F}))<\operatorname{Im}(T)^{B} e^{-2 \pi \operatorname{Im}(T)} .
$$

In other words, the corrections die out exponentially fast as $\operatorname{Im}(T) \rightarrow \infty$.

So in order to understand the behaviour of the periods as $t \rightarrow 0$, it is of interest to know the nilpotent orbit, or equivalently the underlying limiting mixed Hodge structure. Note that the limiting mixed Hodge structure does not sit on the usual cohomology groups of $X_{0}$ (although we saw that it does sit on the logarithmic version of the cohomology groups of $X_{0}$ ). The usual cohomology groups of the central fiber do carry a canonical mixed Hodge structure, but this differs from the limiting mixed Hodge structure. Nevertheless there exists a close relation between them. The precise relation is described by the Clemens-Schmid long exact sequence, which we discuss next.

Let us denote by $H_{\text {lim }}^{k}\left(X_{t}\right)$ the cohomology group of $X_{t}$ equipped with the limiting weight and Hodge filtrations. The Clemens-Schmid sequence is the following long exact sequence:

$$
\ldots \stackrel{\alpha}{\rightarrow} H^{k}\left(X_{0}\right) \stackrel{i^{*}}{\rightarrow} H_{\lim }^{k}\left(X_{t}\right) \stackrel{N}{\rightarrow} H_{\lim }^{k}\left(X_{t}\right) \stackrel{\beta}{\rightarrow} H_{2 n-k}\left(X_{0}\right) \stackrel{\alpha}{\rightarrow} H^{k+2}\left(X_{0}\right) \stackrel{i^{*}}{\rightarrow} \ldots
$$

The maps are as follows. The map $X_{0} \hookrightarrow \mathcal{X}$ is a homotopy equivalence, so we have $H^{*}\left(X_{0}\right) \cong H^{*}(\mathcal{X})$. The inclusion $i: X_{t} \hookrightarrow \mathcal{X}$ yields the restriction map $i^{*}: H^{k}(\mathcal{X}) \rightarrow H^{k}\left(X_{t}\right)$ on cohomology. The map $\beta$ is simply the composition of Poincaré duality on $X_{t}, H^{k}\left(X_{t}\right) \rightarrow H_{2 n-k}\left(X_{t}\right)$, followed by the inclusion $i_{*}$ : $H_{2 n-k}\left(X_{t}\right) \rightarrow H_{2 n-k}(\mathcal{X}) \cong H_{2 n-k}\left(X_{0}\right)$. Of course Poincaré duality on a smooth space is an isomorphism, so the interesting part of this map is $i_{*}$. Finally, the map $\alpha$ is the composition of Poincare duality on the total space $\mathcal{X}, H_{2 n-k}(\mathcal{X}) \rightarrow$ $H^{k+2}(\mathcal{X}, \partial \mathcal{X})$, followed by the natural map $H^{k+2}(\mathcal{X}, \partial \mathcal{X}) \rightarrow H^{k+2}(\mathcal{X})$. All the maps are morphisms of mixed Hodge structures, i.e. morphisms of the underlying lattices which preserves both the Hodge and the weight filtration, with a degree shift on the Hodge type: $\alpha$ has degree $(n+1, n+1), i^{*}$ has degree $(0,0), N$ has degree $(-1,-1)$, and $\beta$ has degree $(-n,-n)$. 
To summarize, given a semi-stable degeneration, we can figure out the behaviour of the periods by taking the following steps: we find the mixed Hodge structure on the central fiber, which can be deduced from a stratification as in equation (A.14); then we use Clemens-Schmid or alternatively the logarithmic cohomology groups to figure out the limiting mixed Hodge structure and the nilpotent orbit; and finally we use the nilpotent orbit theorem to conclude that the latter is a good approximation to the periods.

\section{Appendix B: On the value of the holomorphic Chern-Simons action}

We discussed some aspects of holomorphic Chern-Simons theory in section 3. Here we would like to would like to argue that the value of the holomorphic ChernSimons action evaluates to zero for holomorphic bundles that admit a spectral construction, modulo the usual periodic identifications. We were motivated to write this appendix after a question raised by E. Witten regarding the ChernSimons contribution to the gravitino mass in heterotic string compactifications, which is given by $m_{3 / 2}=e^{\mathcal{K} / 2}\left|W_{0}\right|$.

From the perspective of heterotic/ $F$-theory duality, the reason for the vanishing is very simple. The superpotential of the dual $F$-theory compactification is given by

$$
W_{F}=\frac{1}{2 \pi} \int_{Y} \Omega^{4,0} \wedge \mathrm{G}
$$

The part of the G-flux that comes from the heterotic bundle data is of Hodge type $(2,2)$. The superpotential automatically evaluates to zero on such fluxes. The same argument implies that the $D 7$-brane superpotential does not contribute to the gravitino mass in IIb vacua that can be lifted to $F$-theory.

This argument is not very direct and perhaps somewhat unsatisfactory, since it requires us to invoke duality. We will try to give a more direct and conceptual argument below. The argument below however relies on a construction which is still partly conjectural.

Let us consider a holomorphic $G$-bundle on an elliptically fibered Calabi-Yau three-fold $Z$ with section. We assume that the bundle is semi-stable on generic fibers. Let us temporarily focus our attention on a single elliptic fiber $E$. For 
every semi-stable $G$-bundle on $E$, we can construct a rational surface $R$ containing $E$ as the boundary, and a canonical $G$-bundle $V$ on $R$, such that the restriction $\left.V\right|_{E}$ recovers our $G$-bundle on $E$. This is relatively well-known when $G$ is an exceptional group, in which case $R$ is a del Pezzo surface [59, 63], but it can in fact be done for any gauge group $G$ (see eg. [64]). For the case $G=S O(32)$, this plays an important role in the $S O(32)$ limit of $F$-theory [7].

We can now consider a relative version of this construction, by fibering over a base $B$. We will want to consider the case $\pi: Z \rightarrow B$ where $Z$ is our Calabi-Yau three-fold. Then we should get a holomorphic bundle $V$ on a $\log$ four-fold $(Y, Z)$ which restricts to our original bundle on $Z$. Furthermore, we expect that the twisting data is given by

$$
\frac{\mathrm{G}}{2 \pi}=p_{1}(V)-\frac{1}{2} p_{1}(T Y(-\log Z))
$$

These statements have not been fully shown. The current method is to do a Fourier-Mukai transform along the elliptic fibers and then use a cylinder mapping to construct $Y$ together with a Deligne cohomology class, and this has not been carried out for all groups $G$. When it has, it has not been shown mathematically that the resulting $(2,2)$ flux can be interpreted as above. But we believe that these constructions can be carried out.

Assuming this, we see that our holomorphic bundle on $Z$ can actually be extended to a holomorphic bundle on $(Y, Z)$. By the transgression argument discussed previously in section 3 , we can now directly show that the holomorphic Chern-Simons action can be rewritten as

$$
W_{C S}=\frac{1}{2 \pi} \int_{Y} \Omega^{4,0} \wedge\left(\mathrm{G}-\mathrm{G}_{0}\right)
$$

with the G-flux above. Here we added a reference flux $G_{0}$ such that $G-G_{0}$ restricts to zero in $H^{4}(Z)$. Writing $\mathrm{G}_{0}^{0,4}=\bar{\partial} \omega$, it actually depends only on $\left.\omega\right|_{Z}$. The dependence on a basepoint can be cancelled when we consider the full Chern-Simons action, by tadpole cancellation in the heterotic string, or because the relevant G-flux lives in $\operatorname{ker}\left(d^{4}\right)$ from the point of view of section 4 .

Now since our extended bundle is holomorphic, $G$ is of type $(2,2)$, and so the holomorphic Chern-Simons action evaluates to zero. Conceptually this is the 
holomorphic analogue of a statement in ordinary Chern-Simons theory, which says that the action automatically vanishes if a flat connection on a real threemanifold $M_{3}$ can be extended to a flat connection on $M_{4}$ with $M_{3}=\partial M_{4}$, as one would have $\operatorname{Tr}(F \wedge F)=0$.

The argument may be applied to other examples. Consider for instance a quintic three-fold. It is precisely the $\log$ boundary of $\mathbf{P}^{4}$. So any bundle on the quintic that extends to a holomorphic bundle on $\mathbf{P}^{4}$, or is constructed from such, should have zero Chern-Simons invariant.

One may wonder at this point if one can produce examples with non-zero Chern-Simons invariant, and hence non-zero gravitino mass. At least if one ignores the issue of stability, then it is not hard to do so. Let us simply take a configuration of curves $C_{i}$ on the quintic, and wrap heterotic five-branes on them with multiplicity $n^{i}$, such that $\sum_{i} n^{i}\left[C_{i}\right]$ is a trivial class in homology. Then by classic work of Griffiths and Clemens (see eg. [62]), there are configurations whose Abel-Jacobi map is non-zero, for example the difference of two lines. By a theorem of Voisin, such examples exist on all families of Calabi-Yau three-folds. By varying moduli we can take the projection on the $(0,3)$ component to be non-zero. However such a configuration is clearly not stable as some of the $n^{i}$ have to be taken negative. Producing stable examples appears to be harder.

\section{References}

[1] H. Verlinde and M. Wijnholt, "Building the standard model on a D3-brane," JHEP 0701, 106 (2007) [hep-th/0508089].

[2] R. Donagi and M. Wijnholt, "Higgs Bundles and UV Completion in FTheory," Comm. Math. Phys. 2014, arXiv:0904.1218 [hep-th].

[3] C. Cordova, "Decoupling Gravity in F-Theory," Adv. Theor. Math. Phys. 15, 689 (2011) [arXiv:0910.2955 [hep-th]].

[4] R. Donagi and M. Wijnholt, "Model Building with F-Theory," Adv. Theor. Math. Phys. 15, 1237 (2011) [arXiv:0802.2969 [hep-th]]. 
[5] C. Beasley, J. J. Heckman and C. Vafa, "GUTs and Exceptional Branes in F-theory - I," JHEP 0901, 058 (2009) [arXiv:0802.3391 [hep-th]].

[6] H. Hayashi, R. Tatar, Y. Toda, T. Watari and M. Yamazaki, "New Aspects of Heterotic-F Theory Duality," Nucl. Phys. B 806, 224 (2009) [arXiv:0805.1057 [hep-th]].

[7] A. Clingher, R. Donagi and M. Wijnholt, "The Sen Limit," arXiv:1212.4505 [hep-th].

[8] Y. Kawamata and Y. Namikawa, "Logarithmic deformations of normal crossing varieties and smoothing of degenerate Calabi-Yau varieties," Inventiones Mathematicae, Volume 118, Number 1 (1994), 395-409.

[9] J. Kollár, "Deformations of elliptic Calabi-Yau manifolds," arXiv:1206.5721 [math.AG].

[10] A. Clingher and J. W. Morgan, "Mathematics underlying the F theory / Heterotic string duality in eight-dimensions," Commun. Math. Phys. 254, 513 (2005) [math/0308106 [math-ag]].

[11] R. Donagi, L. Ein and R. Lazarsfeld, "A non-linear deformation of the Hitchin dynamical system," arXiv:alg-geom/9504017.

[12] J. H. M. Steenbrink, "Limits of Hodge structures," Invent. Math. 31, 229-257 (1976).

[13] F. Kato, "Log Smooth Deformation Theory," eprint arXiv:alggeom/9406004, Kyoto-Math 94-07.

[14] W. Schmid, "Variation of Hodge structure: the singularities of the period mapping," Inventiones Math. 22, 211-319 (1973).

[15] W. Fulton, "Intersection Theory," Springer Verlag, Berlin, 1984.

[16] J. Tate, "Algorithm for determining the type of a singular fiber in an elliptic pencil," Modular Functions of One Variable IV, Lecture Notes in Mathematics, vol. 476 (1975), Berlin / Heidelberg: Springer, pp. 33-52. 
[17] M. Bershadsky, K. A. Intriligator, S. Kachru, D. R. Morrison, V. Sadov and C. Vafa, "Geometric singularities and enhanced gauge symmetries," Nucl. Phys. B 481, 215 (1996) [arXiv:hep-th/9605200].

[18] S. Katz, D. R. Morrison, S. Schafer-Nameki and J. Sully, "Tate's algorithm and F-theory," JHEP 1108, 094 (2011) [arXiv:1106.3854 [hep-th]].

[19] M. Esole and R. Savelli, "Tate Form and Weak Coupling Limits in F-theory," arXiv:1209.1633 [hep-th].

[20] R. Donagi and M. Wijnholt, "MSW Instantons," JHEP 1306, 050 (2013), arXiv:1005.5391 [hep-th].

[21] M. F. Atiyah, "Topological Field Theories," Publications mathématiques de l'IHES, 68 (1988), p. 175-186.

[22] E. Witten, "Quantum Field Theory and the Jones Polynomial," Commun. Math. Phys. 121, 351 (1989).

[23] E. Witten, "On quantum gauge theories in two-dimensions," Commun. Math. Phys. 141, 153 (1991).

[24] R. Dijkgraaf and E. Witten, "Topological Gauge Theories and Group Cohomology," Commun. Math. Phys. 129, 393 (1990).

[25] G. W. Moore and N. Seiberg, "Taming the Conformal Zoo," Phys. Lett. B 220, 422 (1989).

[26] D. R. Morrison and C. Vafa, "Compactifications of F theory on Calabi-Yau threefolds. 2.," Nucl. Phys. B 476, 437 (1996) [hep-th/9603161].

[27] P. S. Aspinwall and D. R. Morrison, "Point - like instantons on K3 orbifolds," Nucl. Phys. B 503, 533 (1997) [hep-th/9705104].

[28] S. K. Donaldson and R. P. Thomas, "Gauge theory in higher dimensions," The Geometric Universe: Science, Geometry and the work of Roger Penrose, (SA Huggett et al eds.), Oxford University Press, 1998.

[29] B. Khesin and A. Rosly, "Polar homology and holomorphic bundles," Phil. Trans. Roy. Soc. Lond. A 359, 1413 (2001) [math/0102152]. 
[30] R. P. Thomas, "Gauge Theory on Calabi-Yau Manifolds, Ph.D. Thesis, 1997. R. P. Thomas, "A holomorphic Casson invariant for Calabi-Yau 3-folds, and bundles on K3 fibrations," eprint arXiv:math/9806111, Jour. Diff. Geom. 54, no. 2, 367-438, 2000.

[31] E. Witten, "Branes and the dynamics of QCD," Nucl. Phys. B 507, 658 (1997) [hep-th/9706109].

[32] A. N. Tyurin, "Non-abelian analogues of Abel's theorem", Izv. RAN. Ser. Mat., 65:1 (2001), 133196.

[33] H. Clemens, "Cohomology and Obstructions II: Curves on K-trivial threefolds," eprint arXiv:math/0206219.

[34] D. R. Morrison and J. Walcher, "D-branes and Normal Functions," Adv. Theor. Math. Phys. 13 (2009) [arXiv:0709.4028 [hep-th]].

[35] E. Witten, "On flux quantization in M theory and the effective action," J. Geom. Phys. 22, 1 (1997) [hep-th/9609122].

[36] H. Jockers, P. Mayr and J. Walcher, "On N=1 4d Effective Couplings for F-theory and Heterotic Vacua," Adv. Theor. Math. Phys. 14, 1433 (2010) [arXiv:0912.3265 [hep-th]].

[37] I. B. Frenkel and B. A. Khesin, "Four-dimensional realization of twodimensional current groups," Commun. Math. Phys. 178, 541 (1996).

[38] E. Witten, "Perturbative gauge theory as a string theory in twistor space," Commun. Math. Phys. 252, 189 (2004) [hep-th/0312171].

[39] I. Frenkel and A. Todorov, "Complex Counterpart of Chern-Simons-Witten Theory and Holomorphic Linking," eprint arXiv:math/0502169.

[40] L. J. Mason and D. Skinner, "The Complete Planar S-matrix of N=4 SYM as a Wilson Loop in Twistor Space," JHEP 1012, 018 (2010) [arXiv:1009.2225 [hep-th]].

M. Bullimore and D. Skinner, "Holomorphic Linking, Loop Equations and Scattering Amplitudes in Twistor Space," arXiv:1101.1329 [hep-th]. 
[41] A. Ogus, "Lectures on Logarithmic Algebraic Geometry," 2006.

[42] D. Abramovich et al, "Logarithmic geometry and moduli," Handbook of Moduli, Farkas and Morrison, eds., eprint arXiv:1006.5870.

[43] M. Gross, "Tropical Geometry and Mirror Symmetry," CBMS Regional Conference Series in Mathematics, vol. 114, 2011.

[44] R. Friedman, "Global smoothings of varieties with normal crossings," Annals of Mathematics, 118 (1983), 75-114.

[45] N.-H. Lee, "Constructive Calabi-Yau manifolds," Ph.D. Thesis, The university of Michigan, 2006.

N.-H. Lee, "Hodge numbers of Calabi-Yau manifolds by smoothing of normal crossing varieties," J. of the Chungcheong Mat. Soc., vol. 21, no. 4, 2008.

[46] J. Carlson, "Extensions of mixed Hodge structures," Journées de Géometrie Algébrique d'Angers 1979, Sijthoff and Nordhoff (1980)

J. Carlson, "The geometry of the extension class of a mixed Hodge structure," Proceedings of Symposia in Pure Mathematics 46 (1987).

[47] G. Curio and R. Y. Donagi, "Moduli in N=1 heterotic / F theory duality," Nucl. Phys. B 518, 603 (1998) [hep-th/9801057].

[48] C. Peters and J. Steenbrink, "Mixed Hodge Structures," Springer Verlag, 1998.

[49] P. Berglund and P. Mayr, "Heterotic string / F theory duality from mirror symmetry," Adv. Theor. Math. Phys. 2, 1307 (1999) [hep-th/9811217].

[50] M. Alim, M. Hecht, P. Mayr and A. Mertens, "Mirror Symmetry for Toric Branes on Compact Hypersurfaces," JHEP 0909, 126 (2009) [arXiv:0901.2937 [hep-th]].

[51] M. Alim, M. Hecht, H. Jockers, P. Mayr, A. Mertens and M. Soroush, "Hints for Off-Shell Mirror Symmetry in type II/F-theory Compactifications," Nucl. Phys. B 841, 303 (2010) [arXiv:0909.1842 [hep-th]]. 
[52] H. Jockers and M. Soroush, "Effective superpotentials for compact D5brane Calabi-Yau geometries," Commun. Math. Phys. 290, 249 (2009) [arXiv:0808.0761 [hep-th]].

[53] T. W. Grimm, T. -W. Ha, A. Klemm and D. Klevers, "Computing Brane and Flux Superpotentials in F-theory Compactifications," JHEP 1004, 015 (2010) [arXiv:0909.2025 [hep-th]].

[54] T. W. Grimm, T. -W. Ha, A. Klemm and D. Klevers, "Five-Brane Superpotentials and Heterotic / F-theory Duality," Nucl. Phys. B 838, 458 (2010) [arXiv:0912.3250 [hep-th]].

[55] W. Lerche and S. Stieberger, "Prepotential, mirror map and F theory on K3," Adv. Theor. Math. Phys. 2, 1105 (1998) [Erratum-ibid. 3, 1199 (1999)] [hep-th/9804176].

[56] W. Lerche, "On the heterotic / F theory duality in eight-dimensions," hepth/9910207.

[57] M. Aganagic and C. Beem, "The Geometry of D-Brane Superpotentials," JHEP 1112, 060 (2011) [arXiv:0909.2245 [hep-th]].

[58] R. Blumenhagen, T. W. Grimm, B. Jurke and T. Weigand, "Global F-theory GUTs," Nucl. Phys. B 829, 325 (2010) [arXiv:0908.1784 [hep-th]].

[59] R. Friedman, J. Morgan and E. Witten, "Vector bundles and F theory," Commun. Math. Phys. 187, 679 (1997) [hep-th/9701162].

[60] M. Gross and B. Siebert, "Mirror Symmetry via Logarithmic Degeneration Data I" eprint arXiv:math/0309070.

M. Gross and B. Siebert, "Mirror Symmetry via Logarithmic Degeneration Data II" eprint arXiv:0709.2290[math.AG].

[61] P. A. Griffiths et. al, "Topics in Transcendental Algebraic Geometry," Annals of Mathematics Studies 106, Princeton University Press 1984.

[62] C. Voisin, "Hodge theory and complex algebraic geometry I," Cambridge University Press 2002. 
C. Voisin, "Hodge theory and complex algebraic geometry II," Cambridge University Press 2003.

[63] R. Friedman and J. W. Morgan, "Exceptional groups and del Pezzo surfaces," eprint arXiv:math/0009155.

[64] Conan Leung, Naichung; Zhang, Jiajin, "Moduli of bundles over rational surfaces and elliptic curves I: simply laced cases," arXiv:0906.3900.

Conan Leung, Naichung; Zhang, Jiajin, "Moduli of bundles over rational surfaces and elliptic curves II: non-simply laced cases," arXiv:0908.1645.

Ron Donagi

Department of Mathematics, University of Pennsylvania

Philadelphia, PA 19104-6395, USA

E-mail: donagi@math.upenn.edu

Sheldon Katz

Department of Mathematics, University of Illinois

1409 W. Green St., Urbana, IL 61801, USA

E-mail: katz@math.uiuc.edu

Martijn Wijnholt

Arnold Sommerfeld Center, Ludwig-Maximilians Universität

Theresienstrasse 37, D-80333 München, Germany

E-mail: Martijn.Wijnholt@physik.uni-muenchen.de 